\title{
EXISTENTIALLY EQUIVALENT CYCLIC ULTRAMETRIC SPACES AND CYCLICALLY VALUED GROUPS.
}

\author{
G. LELOUP
}

\begin{abstract}
The notions of ultrametric distances and cyclic valuations appear when the set of values of the distance map is a cyclically ordered set. These structures can be described as subspaces of cartesian products. In this paper we characterize existentially equivalence between cyclically ultrametric spaces, as well as existentially equivalence between generalized ultrametric spaces. We also describe classes of existentially equivalent cyclically valued groups.
\end{abstract}

\section{INTRODUCTION.}

A cyclically ordered set is a set $C$ equipped with a ternary relation $(\cdot, \cdot, \cdot)$ that satisfies $(1),(2),(3)$ below.

(1) $\forall a, b, c,(a, b, c) \Rightarrow a \neq b \neq c \neq a$

(2) $\forall a, b, c,(a, b, c) \Rightarrow(b, c, a)$

(3) $\forall a \in C,(a, \cdot, \cdot)$ is a total order on $C \backslash\{a\}$

(see [Fu 63] and [No 82]).

For every $a \in C$, let $\leq_{a}$ be the total order on $C$ defined by: $x \leq_{a} y \Leftrightarrow$ either $x=a$ or $(a, x, y)$ or $x=y$. For $\varnothing \neq S \subset C, \min _{a} S$ will denote the minimum (if any) of $\left(S, \leq_{a}\right)$.

Observe that any totally ordered set $(T, \leq)$ can be equipped with a canonic cyclic order by setting: $\forall a, b, c,(a, b, c) \Leftrightarrow$ either $a<b<c$ or $b<c<a$ or $c<a<b$. We will say that $(\cdot, \cdot, \cdot)$ is the cyclic order defined by $\leq$.

Let $S$ be a subset of $C$. The reader will easily prove that the following (i), (ii) are equivalent.

(i) There exists $a \in C$, such that the totally ordered set $\left(S, \leq_{a}\right)$ is well-ordered.

(ii) For every $a \in C$, the totally ordered set $\left(S, \leq_{a}\right)$ is well-ordered.

If this holds, we will say that $(S,(\cdot, \cdot, \cdot))$ is well-ordered.

In [GKL], $C$ was assumed to be a group, and cyclically valued rings were defined. Such rings are equipped with "valuations" with values in $C$. Characterizing elementary equivalent cyclically valued rings is still an open question. In the present paper, we consider similar problem with a language without a multiplication symbol. This way we obtain cyclically valued groups. We also consider a language with a symbol interpreted by the distance defined by the valuation. This gives rise to cyclically ultrametric space. First we review some properties of these structures.

In the following, $C$ is a cyclically ordered set.

Let $\infty \notin C$, and we extend the order $\leq_{a}$, with $a \in C$, to $C \cup\{\infty\}$ by setting: $\forall a \in C, \forall b \in C, b \leq_{a} \infty$.

Definition 1.1. Let $E$ be a non-empty set and $u$ be a mapping from $C \times E \times E$ to $C \cup\{\infty\}$. For $a \in C$, $u_{a}=u(a, \cdot, \cdot)$ will denote the restriction of $u$ to $\{a\} \times E \times E$. We will say that $(E, u, C)$ is a cyclically ultrametric space if, for every $a \in C,\left(E, u_{a}\right)$ is an ultrametric space, where $C$ is equipped with the order $\leq a$ (that is:

$\forall \sigma \in E, \forall \tau \in E, u(a, \sigma, \tau)=u(a, \tau, \sigma)$,

$\forall \sigma \in E, \forall \tau \in E,(u(a, \sigma, \tau)=\infty \Leftrightarrow \sigma=\tau)$,

$\left.\forall \sigma \in E, \forall \tau \in E, \forall \rho \in E, u(a, \sigma, \tau) \geq_{a} \min _{a}(u(a, \sigma, \rho), u(a, \rho, \tau))\right)$.

Definition 1.2. Let $G$ be an abelian group and $v$ be a mapping from $C \times G$ to $C \cup\{\infty\}$. For $a \in C$, $v(a, \cdot)$ will denote the restriction of $v$ to $\{a\} \times G$.

We will say that $(G,+, v)$ is a cyclically valued group if for every $a \in C,(G,+, v(a, \cdot))$ is a valued group,

2000 Mathematics Subject Classification. Primary 03 C 65, 06 F 15, 06 F 99, 20 F 99; Secondary 06 A 99.

Key words and phrases. Existentially equivalent, cyclically ordered sets, ultrametric spaces, valued groups. 
where $C$ is equipped with the order $\leq_{a}$ (that is:

$\forall \sigma \in G,(v(a, \sigma)=\infty \Leftrightarrow \sigma=0)$

$\left.\forall \sigma \in G, \forall \tau \in G, v(a, \sigma-\tau) \geq_{a} \min _{a}(v(a, \sigma), v(a, \tau))\right)$.

For $\sigma, \tau$ in $E, E$ a cyclically ultrametric space, the support of $\sigma, \tau$ is the $\operatorname{set} \operatorname{Supp}(\sigma, \tau):=\{u(a, \sigma, \tau) \mid a \in$ $C\} \cap C(\operatorname{Supp}(\sigma, \sigma)=\varnothing)$.

We will say that $u$ is support-definable if for every $a$ in $C$ and $\sigma, \tau$ in $E, \min _{a} \operatorname{Supp}(\sigma, \tau)$ exists and is equal to $u(a, \sigma, \tau)$. In Subsection 2.1, we define a canonical embedding, from any cyclically ultrametric space such that $u$ is support-definable, into some cartesian products (Theorem 1).

Let $(G,+, v)$ be a cyclically valued group. For every $a \in C, \sigma \in G, \tau \in G$, we set $u(a, \sigma, \tau)=v(a, \sigma-\tau)$. We will say that $v$ is support-definable if $u$ is support-definable. In Subsection 2.2, we get an analog of Theorem 1 in the case of cyclically valued groups (see Theorem 2).

Section 3 is devoted to model theoretic properties of cyclic distances and of cyclic valuations. We define rich cyclically ultrametric spaces (following the terminology of [D 84]) and show that every cyclically ultrametric space admits a "rich closure". Next, thanks to the use of an appropriate language (and with additionnal conditions), we prove that any two rich cyclically ultrametric spaces are existentially equivalent (Theorem 3).

We can use that same language to study generalized ultrametric spaces, so, in Subsection 3.3, we get classes of extistentially equivalent generalized ultrametric spaces.

We also define rich cyclically valued groups, rich closures (Subsection 3.5), and existentially equivalent rich cyclically valued groups (Theorem 4).

In Section 4, we prove that the cyclic ultrametric distances and the cyclic valuations are more general than the classical ones. Indeed, for every ultrametric space $(E, \nu)$, there exists a cyclic ultrametric distance $u$ on $E$ such that $\nu$ is the ultrametric distance defined by a Dedekind cut of $C$ (Theorem 5). In the same way, for every valued group $(G,+, \nu)$, there exists a cyclic valuation $v$ on $G$ such that $\nu$ is the ultrametric distance defined by a Dedekind cut of $C$ (Theorem 6 ).

In Section 5, having fixed an element $O$ in $E$, we define monomials as being the elements $\mu$ of $E$ such that $\operatorname{Supp}(\mu, O)$ is a singleton. Then, the single element of $\operatorname{Supp}(\mu, O)$ will be called the degree of $\mu$. We will say that the set of monomials u-represents $E$ if $u$ is support-definable and, for every $a$ in $C$ and $\sigma$ in $E$ such that $u(a, \sigma, O)=a$, there exists a monomial $\mu$ such that $u(a, \sigma, \mu) \neq a$. We will prove that, if the set of monomials $u$-represents $E$, then $E$ embeds in $\prod_{a \in C}\left(M_{a} \cup\{O\}\right)$, where $M_{a}$ is the set of all monomials of degree $a$ (Theorem 7).

Let $(G,+, v)$ be a cyclically valued group. $(G, 0, u)$ is a cyclically ultrametric space with 0 as a fixed element. In this case, the 0 -monomials will be called monomials. We will set $\operatorname{Supp}(\sigma):=\operatorname{Supp}(\sigma, 0)$. We will say that the set of monomials v-represents $G$ if the set of monomials $u$-represents the set $G$. We get an analog of Theorem 7 in the case of cyclically valued groups (see Theorem 8).

\section{SupporT-DEFINABILITY.}

2.1. Cyclically ultrametric spaces. We will denote by $\mathcal{L B}$ the set $\{\varnothing\} \cup\left\{S \subset C \mid \forall a \in C, \min _{a} S\right.$ exists $\}$. We see that $(\mathcal{L B}, \subset)$ is an ordered set. It is a join-semilattice, but it needs not be a lattice.

Let $(E, u, C)$ be a cyclically ultrametric space. Another way of saying that $u$ is support-definable is: for every $\sigma$ and $\tau$ in $E, \operatorname{Supp}(\sigma, \tau) \in \mathcal{L B}$, and, for every $a$ in $C, u(a, \sigma, \tau)=\min _{a} \operatorname{Supp}(\sigma, \tau)$ (if $\sigma=\tau$, we set $\left.\min _{a} \operatorname{Supp}(\sigma, \tau):=\infty\right)$.

We will sometimes use the following characterization for being support-definable.

Lemma 2.1. Let $(E, u, C)$ be a cyclically ultrametric space. Then $u$ is support-definable if and only if for all $\sigma, \tau$ in $E$, and for all $a, b$ in $C, a \leq_{a} b \leq_{a} u(a, \sigma, \tau) \Rightarrow u(b, \sigma, \tau)=u(a, \sigma, \tau)$.

Proof. Assume that $u$ is support-definable, and let $a$ in $C, \sigma, \tau$ in $E$ such that $a \neq u(a, \sigma, \tau)$. We have $u(a, \sigma, \tau)=\min _{a} \operatorname{Supp}(\sigma, \tau)$, hence: $\forall b, a \leq_{a} b<_{a} u(a, \sigma, \tau) \Rightarrow b \notin \operatorname{Supp}(\sigma, \tau)$. Therefore $\forall b, a \leq_{a}$ $b<_{a} u(a, \sigma, \tau) \Rightarrow u(a, \sigma, \tau)=\min _{b} \operatorname{Supp}(\sigma, \tau)=u(b, \sigma, \tau)$. Clearly, if $b=u(a, \sigma, \tau)$, then $u(b, \sigma, \tau)=$ 
$\min _{b} \operatorname{Supp}(\sigma, \tau)$.

Conversely, assume that for every $a, b$ in $C, a \leq_{a} b \leq_{a} u(a, \sigma, \tau) \Rightarrow u(b, \sigma, \tau)=u(a, \sigma, \tau)$. Thus, for every $b \in C, b \in \operatorname{Supp}(\sigma, \tau) \Leftrightarrow b=u(b, \sigma, \tau)$. Then, for every $a \in C, u(a, \sigma, \tau)=\min _{a} \operatorname{Supp}(\sigma, \tau)$. Hence $\operatorname{Supp}(\sigma, \tau) \in \mathcal{L B}$.

Lemma 2.2. Let $(E, u, C)$ be a cyclically ultrametric space such that $u$ is support-definable, let a in $C$ and $\sigma, \tau, \rho$ in $E$. Then:

a) $a \in \operatorname{Supp}(\sigma, \tau) \Leftrightarrow u(a, \sigma, \tau)=a$,

b) $\operatorname{Supp}(\sigma, \tau) \subset \operatorname{Supp}(\sigma, \rho) \cup \operatorname{Supp}(\rho, \tau)$.

Proof. .

a): trivial.

b) If $u(a, \sigma, \tau)=a$ and $u(a, \rho, \tau) \neq a$, then $u(a, \sigma, \rho)=a$. Now, we deduce from a): $\operatorname{Supp}(\sigma, \tau) \subset$ $\operatorname{Supp}(\sigma, \rho) \cup \operatorname{Supp}(\rho, \tau)$.

Observe that if $u$ is support-definable, then for every $\sigma, \tau$ in $E$ and $a$ in $\operatorname{Supp}(\sigma, \tau)$, the subset $I:=\{b \neq a \mid u(b, \sigma, \tau)=a\}$ is a final segment of $\left(C \backslash\{a\}, \leq_{a}\right)$. Indeed, let $b \in I$ and $c \in C$ with $b<_{a} c$. According to general properties of cyclically ordered sets, we have $c<_{b} a$. By Lemma $2.1, u(c, \sigma, \tau)=a$. Hence $c \in I$.

Proposition 2.3. Let $\left(\Gamma_{a}\right)_{a \in C}$ be a family of non-empty sets and $E$ be a subset of the cartesian product $\prod_{a \in C} \Gamma_{a}$ such that:

$\left(^{*}\right) \forall a \in C, \forall \sigma \in E, \forall \tau \in E \backslash\{\sigma\}, \min _{a}\left(\left\{a \in C \mid \sigma_{a} \neq \tau_{a}\right\}\right)$ exists.

$\min _{a}\left(\left\{a \in C \mid \sigma_{a} \neq \tau_{a}\right\}\right)$ will be denoted by $u(a, \sigma, \tau)$. If $\sigma=\tau$, we set $u(a, \sigma, \tau):=\infty$.

Then $(E, u, C)$ is a cyclically ultrametric space such that $u$ is support-definable.

Proof. Let $a \in C, \sigma \in E, \tau \in E$. It is an immediate consequence of the definition that $u(a, \sigma, \tau)=$ $u(a, \tau, \sigma)$ and $u(a, \sigma, \tau)=\infty \Leftrightarrow \sigma=\tau$.

Now let $a \in C, \sigma, \tau, \rho$ in $E$. Set $b:=\min _{a}(u(a, \sigma, \rho), u(a, \rho, \tau))$.

By assumption, for every $c$ such that $a \leq_{a} c<_{a} b$, we have $\sigma_{c}=\rho_{c}$, and $\rho_{c}=\tau_{c}$. Hence $\sigma_{c}=\tau_{c}$, so $u(a, \sigma, \tau) \geq_{a} b$. Therefore $(E, u, C)$ is a cyclically ultrametric space. Now, we see that the support of $\sigma, \tau$ is $\left\{a \in C \mid \sigma_{a} \neq \tau_{a}\right\}$ and that $u$ is support-definable.

Theorem 1. Let $(E, u, C)$ be a cyclically ultrametric space such that $u$ is support-definable.

For every $a \in C$ and $\sigma \in E$, we set $\sigma_{a}:=\{\tau \in E \mid u(a, \sigma, \tau) \neq a\} \subset E$, and we let $\Gamma_{a}$ be the set of all the $\sigma_{a}$ 's. Then:

(a) for every a in $C, \sigma, \tau$ in $E, u(a, \sigma, \tau)=\min _{a}\left\{b \in C \mid \sigma_{b} \neq \tau_{b}\right\}$.

(b) the mapping $\sigma \mapsto\left(\sigma_{a}\right)_{a \in C}$ is an isomorphism from the cyclically ultrametric space $(E, u, C)$ into a subset of $\prod_{a \in C} \Gamma_{a}$ which satisfies condition $\left(^{*}\right)$ of Proposition 2.3.

Proof. For every $a \in C$, the relation: $u(a, \sigma, \tau) \neq a$ is an equivalence relation on $E$. Hence: $\sigma_{a}=\tau_{a} \Leftrightarrow$ $u(a, \sigma, \tau) \neq a$.

Let $a$ in $C, \sigma, \tau$ in $E$ and $c:=u(a, \sigma, \tau)$. By Lemma 2.2 a), $c=u(c, \sigma, \tau)$, hence $\sigma_{c} \neq \tau_{c}$. Let $b \in C$ such that $a \leq_{a} b<_{a} c$. Then $u(b, \sigma, \tau)=c \neq b$, hence $\sigma_{b}=\tau_{b}$. Thus $c=\min _{a}\left\{b \in C \mid \sigma_{b} \neq \tau_{b}\right\}$. For every $\sigma \in E$, set $\psi(\sigma):=\left(\sigma_{a}\right)_{a \in C} \in \prod_{a \in C} \Gamma_{a}$. So $\psi(E)$ satisfies condition (*) of Proposition 2.3, and $\forall(\sigma, \tau) \in E \times E, u(a, \sigma, \tau)=u(a, \psi(\sigma), \psi(\tau))$.

2.2. Cyclically valued groups. In the same way as Proposition 2.3, we can prove:

Proposition 2.4. Let $\left(\Gamma_{a}\right)_{a \in C}$ be a family of non-trivial abelian groups, $G$ be a subgroup of the cartesian product $\prod_{a \in C} \Gamma_{a}$ such that:

(*) $\forall a \in C, \forall \sigma \in G, \min _{a}\left(\left\{a \in C \mid \sigma_{a} \neq 0\right\}\right)$ exists.

$\min _{a}\left(\left\{a \in C \mid \sigma_{a} \neq 0\right\}\right)$ will be denoted by $v(a, \sigma)$. If $\sigma=0$, we set $v(a, \sigma):=\infty$.

Then $(G,+, v)$ is a cyclically valued group such that $v$ is support-definable.

Let $(G,+, v)$ be a cyclically valued group such that $v$ is support-definable. For every $a$ and $b$ in $C$, the sets $G_{a}:=\{\sigma \in G \mid v(a, \sigma) \neq a\}$ are subgroups of $G$. Let $\psi_{a}$ be the canonical mapping from $G$ onto $G / G_{a}$. 
Theorem 2. Let $(G,+, v)$ be a cyclically valued group such that $v$ is support-definable. For every a $\in C$, set $\Gamma_{a}:=G / G_{a}$, and for every $\sigma \in G$, set $\psi(\sigma):=\left(\psi_{a}(\sigma)\right)_{a \in C}$.

Then $\psi$ is an isomorphism from the cyclically valued group $(G,+, v)$ onto a subgroup of $\prod_{a \in C} \Gamma_{a}$ which satisfies condition (*) of Proposition 2.4.

Proof. First of all, note that, for every $\sigma \in G$ and $a \in C$, we have: $\psi_{a}(\sigma) \neq \psi_{a}(0) \Leftrightarrow a \in \operatorname{Supp}(\sigma)$. Hence $\operatorname{Supp}(\psi(\sigma))=\operatorname{Supp}(\sigma)$, and $\psi(G)$ satisfies condition $\left(^{*}\right)$ of Proposition 2.4.

Let $\sigma \neq \tau$ in $G$ and $a \in \operatorname{Supp}(\sigma-\tau)$. So $v(a, \sigma-\tau) \neq a$, hence $\psi_{a}(\sigma) \neq \psi_{a}(\tau)$. It follows that $\psi$ is one-to-one.

$\psi$ is trivially a homomorphism from the group $(G,+)$ to $\prod_{a \in C} \Gamma_{a}$. It remains to prove that, for all $\sigma \in G$ and $a \in C, v(a, \sigma)=v(a, \psi(\sigma))$.

Assume that $v(a, \sigma)=a$. Then $\psi_{a}(\sigma) \neq \psi_{a}(0)$, hence $v(a, \psi(\sigma))=a$.

Assume that $v(a, \sigma)=b \neq a$. Since $v$ is support-definable, it follows from Lemma 2.1 that, for every $c \in C$ such that $a \leq_{a} c<_{a} b$, we have: $v(c, \sigma)=b \neq c$. Hence for every $c \in C$ with $a \leq_{a} c<_{a} b$, $\psi_{c}(\sigma)=\psi_{c}(0)$. Now, $v(b, \sigma)=b$. Consequently $\psi_{b}(\sigma) \neq \psi_{b}(0)$. Therefore $v(a, \psi(\sigma))=b$.

Let $\left(\Gamma_{a}\right)_{a \in C}$ be a family of abelian groups. The subset of all elements $\sigma$ of $\prod_{a \in C} \Gamma_{a}$, such that, for every $a$ in $C, \min _{a} \operatorname{Supp}(\sigma) \backslash\{a\}$ exists, needs not be a subgroup of the cartesian product $\prod_{a \in C} \Gamma_{a}$. For example, let $C$ be the cyclically ordered set $\mathbb{Z}$ of all integers, and for every $n \in \mathbb{Z}$, let $\Gamma_{a}$ be the additive group $\mathbb{Q}$ of all rational numbers. Set $\sigma_{1}:=\sum_{n \in \mathbb{Z}} X^{n}$ and $\sigma_{2}:=\sum_{n \in \mathbb{N}} X^{n}$. For every $n$ in $\mathbb{Z}$, $\min _{a}\left(\operatorname{Supp}\left(\sigma_{1}\right)\right)$ and $\min _{a}\left(\operatorname{Supp}\left(\sigma_{2}\right)\right)$ exist, but $\left(\operatorname{Supp}\left(\sigma_{1}-\sigma_{2}\right),<_{1}\right)$ has no lowest element.

However, $\prod_{a \in C} \Gamma_{a}$ contains subgroups which satisfy condition (*) of Proposition 2.4: for example the subgroup of all elements whose supports are both finite or empty, and the subgroup $\sqcap_{a \in C} \Gamma_{a}$ of all elements with well-ordered support, that we will call the Hahn product.

As another example, let $C$ be the cyclically ordered set $\mathbb{Z}$ and for every $a \in C$, set $\Gamma_{a}:=\mathbb{Z}$. For every prime number $p$, we let $\sigma^{p}$ be the element of $\prod_{a \in C} \Gamma_{a}$ such that $\sigma_{i}^{p}=1$ if and only if $i \in p \mathbb{Z}$, and $\sigma_{i}^{p}=0$ otherwise. Now, we let $G$ be the subgroup of $\prod_{a \in C} \Gamma_{a}$ generated by all the $\sigma^{p}$, where $p$ is a prime number, and by the elements whose supports are finite. In order to prove that $G$ satisfies condition $(*)$ of Proposition 2.4, we let $\sigma=n_{1} \sigma^{p_{1}}+\cdots+n_{k} \sigma^{p_{k}}+\rho \in G$, where $n_{i} \in \mathbb{Z}(1 \leq i \leq k)$ and $\operatorname{Supp}(\rho)$ is finite. Now, let $m \in \operatorname{Supp}(\sigma)$. The proof will be completed by showing that $\operatorname{Supp}(\sigma)$ contains an element greater than $m$ (with $<$ the usual order in $\mathbb{Z}$ ). We let $q$ be a prime number such that $q>m$, $q>\operatorname{Supp}(\rho)$ and $q>p_{j}(1 \leq j \leq k)$. Then for every $j(1 \leq j \leq k), q p_{j} \in \operatorname{Supp}\left(\sigma^{p_{j}}\right), q p_{j} \notin \operatorname{Supp}(\rho)$ and $q p_{j} \notin \operatorname{Supp}\left(\sigma^{p_{i}}\right)(i \neq j)$. Thus $q p_{j} \in \operatorname{Supp}(\sigma)$.

\section{MOdel THEORY.}

3.1. Theories of families of equivalence relations. Let $(E, u, C)$ be a cyclically ultrametric space such that $u$ is defined by the supports. For every $a$ in $C$ and $\sigma, \tau$ in $E$, we set $r(a, \sigma, \tau)$ if and only if $u(a, \sigma, \tau) \neq a$. It follows readily that, for each $a \in C, r(a, \cdot, \cdot)$ is a relation of equivalence. The converse can be stated as follows.

Lemma 3.1.1. Let $E$ be a nonempty set, and $r$ be a ternary relation defined on $C \times E \times E$, such that, for every $a \in C, r(a, \cdot, \cdot)$ is an equivalence relation. Assume that for every $a \in C$ and every $\sigma \neq \tau$ in $E, \min _{a}(C \backslash\{a \in C \mid r(a, \sigma, \tau)\})$ exists. We set $u(a, \sigma, \tau):=\min _{a}(C \backslash\{a \in C \mid r(a, \sigma, \tau)\})$, and $u(a, \sigma, \sigma):=\infty$. Then:

1) $(E, u, C)$ is a cyclically ultrametric space such that $u$ is support-definable,

2) for every $a \in C$ and every $\sigma, \tau$ in $E, r(a, \sigma, \tau) \Leftrightarrow u(a, \sigma, \tau) \neq a$.

Proof. Let $a \in C$ and $\sigma, \rho, \tau$ in E. Trivially, $\neg r(a, \sigma, \tau) \Rightarrow(\neg r(a, \sigma, \rho) \vee \neg r(a, \rho, \tau))$. Therefore $(C \backslash\{a \in C \mid r(a, \sigma, \tau)\}) \subset(C \backslash\{a \in C \mid r(a, \sigma, \rho)\}) \cup(C \backslash\{a \in C \mid r(a, \rho, \tau)\})$. Hence $u(a, \sigma, \tau) \geq_{a}$ $\min _{a}(u(a, \sigma, \rho), u(a, \rho, \tau))$. Consequently $(E, u, C)$ is a cyclically ultrametric space.

It follows from hypothesis that: $u(a, \sigma, \tau) \neq a \Leftrightarrow a \notin\{b \in C \mid \neg r(b, \sigma, \tau)\} \Leftrightarrow r(a, \sigma, \tau)$, and $\operatorname{Supp}(\sigma, \tau)=\{a \in C \mid u(a, \sigma, \tau)=a\}$. Hence $u(a, \sigma, \tau)=\min _{a}(C \backslash\{a \in C \mid r(a, \sigma, \tau)\})=\min _{a} \operatorname{Supp}(\sigma, \tau)$. So $u$ is support-definable.

Let both of $I$ and $E$ be nonempty sets. Assume that for each $a$ in $I$, there exists an equivalence relation $r(a, \cdot, \cdot)$ on $E$. That will be denoted by $(E,\{r(a, \cdot, \cdot) \mid a \in I\})$. For every $\sigma, \tau$ in $E$, the support of $(\sigma, \tau)$ will be the set $\operatorname{Supp}(\sigma, \tau):=(I \backslash\{a \in I \mid r(a, \sigma, \tau)\})$, and we set $\sigma \sim \tau$ if and only if $\operatorname{Supp}(\sigma, \tau)=\varnothing$. 
For example, let $I$ be a nonvoid set, and for every $a \in I$ let $\Gamma_{a}$ be a nonvoid set. We let $E$ be $\prod_{a \in I} \Gamma_{a}$, and for every $b \in I, \sigma=\left(\sigma_{a}\right)_{a \in I}, \tau=\left(\tau_{a}\right)_{a \in I}$ in $E$, we set $r(b, \sigma, \tau)$ if and only if $\sigma_{b}=\tau_{b}$.

Let $(E,\{r(a, \cdot, \cdot) \mid a \in I\})$ be a set together with a family of equivalence relations. One can check the following 1), 2), 3).

1) $\sim$ is an equivalence relation.

2) For every $a \in I$, we can define an equivalence relation $r(a, \cdot, \cdot)$ on $E / \sim$.

3) For every $a \in I$, set $\Gamma_{a}:=E / r(a, \cdot, \cdot)$. Then there is a natural embedding from $(E / \sim,\{r(a, \cdot, \cdot) \mid a \in$ $I\})$ into $\left(\prod_{a \in I} \Gamma_{a},\{r(a, \cdot, \cdot) \mid a \in I\}\right)$.

The language $\mathcal{L}$ rel $_{I}$ will be the language $(r(a, \cdot, \cdot), a \in I)$.

For every finite subset $\left\{a_{1}, \ldots, a_{n}, a_{n+1}, \ldots, a_{n+p}\right\}$ of $I$ and every $p$-uple $\left\{i_{1}, \ldots, i_{p}\right\}$ of positive integers,

$$
\Phi_{a_{1}, \ldots, a_{n} ; a_{n+1}, \ldots, a_{n+p} ; i_{1}, \ldots, i_{p}}\left(\sigma_{1}, \ldots, \sigma_{n}, \tau_{1,1}, \ldots, \tau_{1, i_{1}}, \ldots, \tau_{p, 1}, \ldots, \tau_{p, i_{p}}\right)
$$

will be the formula

$$
\begin{gathered}
\exists \tau_{1}, \ldots, \exists \tau_{p}, \neg r\left(a_{n+1}, \tau_{1}, \tau_{1,1}\right), \ldots, \neg r\left(a_{n+1}, \tau_{1}, \tau_{1, i_{1}}\right), \ldots, \\
\neg r\left(a_{n+p}, \tau_{p}, \tau_{p, 1}\right), \ldots, \neg r\left(a_{n+p}, \tau_{p}, \tau_{p, i_{p}}\right) \Rightarrow \\
\exists \sigma, r\left(a_{1}, \sigma_{1}, \sigma\right), \ldots, r\left(a_{n}, \sigma_{n}, \sigma\right), \neg r\left(a_{n+1}, \sigma, \tau_{1,1}\right), \ldots, \neg r\left(a_{n+1}, \sigma, \tau_{1, i_{1}}\right), \ldots, \\
\neg r\left(a_{n+p}, \sigma, \tau_{p, 1}\right), \ldots, \neg r\left(a_{n+p}, \sigma, \tau_{p, i_{p}}\right) .
\end{gathered}
$$

Definition 3.1.2. We will say that $(E,\{r(a, \cdot, \cdot) \mid a \in I\})$ is rich if and only if for every formula

$$
\Phi_{a_{1}, \ldots, a_{n} ; a_{n+1}, \ldots, a_{n+p} ; i_{1}, \ldots, i_{p}}, \text { with } \operatorname{card}\left(\left\{a_{1}, \ldots, a_{n}, a_{n+1}, \ldots, a_{n+p}\right\}\right)=n+p,
$$

$(E,\{r(a, \cdot, \cdot) \mid a \in I\})$ enjoys

$$
\begin{gathered}
\forall \sigma_{1}, \ldots, \forall \sigma_{n}, \forall \tau_{1,1}, \ldots, \forall \tau_{1, i_{1}}, \ldots, \forall \tau_{p, 1}, \ldots, \forall \tau_{p, i_{p}}, \\
\Phi_{a_{1}, \ldots, a_{n} ; a_{n+1}, \ldots, a_{n+p} ; i_{1}, \ldots, i_{p}}\left(\sigma_{1}, \ldots, \sigma_{n}, \tau_{1,1}, \ldots, \tau_{1, i_{1}}, \ldots, \tau_{p, 1}, \ldots, \tau_{p, i_{p}}\right) .
\end{gathered}
$$

For example $\prod_{a \in I} \Gamma_{a}$ is rich.

Proposition 3.1.3. Let $(E,\{r(a, \cdot, \cdot) \mid a \in I\})$ be a set together with a family of equivalence relations. Then, there exists a set $F$, with $E \subset F$, and for every $a \in I$, an extension of $r(a, \cdot, \cdot)$ to $F$ such that:

- $(F,\{r(a, \cdot, \cdot) \mid a \in I\})$ is rich,

- for every $\sigma \in F$, there exists $\tau \in E$ such that $\operatorname{Supp}(\sigma, \tau)$ is finite.

Furthermore, $(F / \sim) \hookrightarrow \prod_{a \in I} E / r(a, \cdot, \cdot)$. If every equivalence class under $\sim$ is a singleton, then we can assume that $F$ embeds in $\prod_{a \in I} E / r(a, \cdot, \cdot)$.

Proof. Let $E^{\prime}$ be a set of representatives of all equivalence class under $\sim$. For every $a \in I$, we let $\Gamma_{a}$ be the quotient set $E / r(a, \cdot, \cdot)$. Then $E^{\prime}$ embeds in a natural way in $\prod_{a \in I} \Gamma_{a}$. Hence we assume that $E^{\prime}$ is a subset of $\prod_{a \in I} \Gamma_{a}$. Now, we set $H:=\prod_{a \in I} \Gamma_{a} \cup\left(E \backslash E^{\prime}\right)$. Notice that $E \subset H$. Let $a_{0} \in I$ and $\sigma, \tau$ in $H$.

If $\sigma$ and $\tau$ belong to $\left(\prod_{a \in I} \Gamma_{a}\right) \backslash E$, we set $r\left(a_{0}, \sigma, \tau\right) \Leftrightarrow \sigma_{a_{0}}=\tau_{a_{0}}$.

If $\sigma \in\left(\prod_{a \in I} \Gamma_{a}\right) \backslash E$ and $\tau \in E$, then there exists $\tau^{\prime} \in E^{\prime}$ such that $\tau \sim \tau^{\prime}$. We set $r\left(a_{0}, \sigma, \tau\right) \Leftrightarrow \sigma_{a_{0}}=$ $\tau_{a_{0}}^{\prime}$. Hence, if $\sigma$ and $\tau$ belong to $\prod_{a \in I} \Gamma_{a}$, then we have $r\left(a_{0}, \sigma, \tau\right) \Leftrightarrow \sigma_{a_{0}}=\tau_{a_{0}}$.

Straightforward checking shows that $r\left(a_{0}, \cdot, \cdot\right)$ is a relation of equivalence and that $H / \sim=\prod_{a \in I} \Gamma_{a}$. Furthermore, $(H,\{r(a, \cdot, \cdot) \mid a \in I\})$ is rich.

Now, we define $F$ to be the union of the sets $E_{t}, t<\omega$, which we construct inductively as follows. We set $E_{0}:=E$. Let $t$ be a non negative integer, and assume that $E_{t}$ is already defined. For every pairwise distinct $a_{1}, \ldots, a_{n}, a_{n+1}, \ldots, a_{n+p}$ in $I, i_{1}, \ldots, i_{p}$ integers, and

$$
\begin{gathered}
\sigma_{1}, \ldots, \sigma_{n}, \tau_{1,1}, \ldots, \tau_{1, i_{1}}, \ldots, \tau_{p, 1}, \ldots \tau_{p, i_{p}} \text { in } E_{t} \text { such that } \exists \tau_{1} \in E_{t}, \ldots, \exists \tau_{p} \in E_{t}, \\
\neg r\left(a_{n+1}, \tau_{1}, \tau_{1,1}\right), \ldots, \neg r\left(a_{n+1}, \tau_{1}, \tau_{1, i_{1}}\right), \ldots, \neg r\left(a_{n+p}, \tau_{p}, \tau_{p, 1}\right), \ldots, \neg r\left(a_{n+p}, \tau_{p}, \tau_{p, i_{p}}\right) \text { and } \\
E_{t}=\neg \Phi_{a_{1}, \ldots, a_{n} ; a_{n+1}, \ldots, a_{n+p} ; i_{1}, \ldots, i_{p}}\left(\sigma_{1}, \ldots, \sigma_{n}, \tau_{1,1}, \ldots, \tau_{1, i_{1}}, \ldots, \tau_{p, 1}, \ldots, \tau_{p, i_{p}}\right),
\end{gathered}
$$

we define $\sigma \in \prod_{a \in I} \Gamma_{a}$ in the following way.

If $a \notin\left\{a_{2}, \ldots, a_{n}, a_{n+1}, \ldots, a_{n+p}\right\}$, then $\sigma_{a}:=\sigma_{1 a}$. For $i, 2 \leq i \leq n$, we set $\sigma_{a_{i}}:=\sigma_{i a_{i}}$, and for $j$, $1 \leq j \leq p, \sigma_{a_{n+j}}:=\tau_{j a_{n+j}}$. Then $\sigma$ satisfies

$$
\begin{gathered}
r\left(a_{1}, \sigma_{1}, \sigma\right), \ldots, r\left(a_{n}, \sigma_{n}, \sigma\right), \neg r\left(a_{n+1}, \sigma, \tau_{1,1}\right), \ldots, \neg r\left(a_{n+1}, \sigma, \tau_{1, i_{1}}\right), \ldots, \neg r\left(a_{n+p}, \sigma, \tau_{p, 1}\right), \ldots, \\
\neg r\left(a_{n+p}, \sigma, \tau_{p, i_{p}}\right),
\end{gathered}
$$


and $\operatorname{Supp}\left(\sigma, \sigma_{1}\right) \subset\left\{a_{1}, \ldots, a_{n}, a_{n+1}, \ldots, a_{n+p}\right\}$. We let $E_{t+1}$ be the union of $E_{t}$ and of the set of all the $\sigma$ 's. Then $E_{t} \subset H$. By construction, $F$ is rich. Let $\sigma \in F$. There exists an integer $t$ and $\tau_{t} \in E_{t}, \tau_{t-1} \in E_{t-1}, \ldots, \tau_{0} \in E_{0}$ such that $\operatorname{Supp}\left(\sigma, \tau_{t}\right), \operatorname{Supp}\left(\tau_{t}, \tau_{t-1}\right), \ldots, \operatorname{Supp}\left(\tau_{1}, \tau_{0}\right)$ are finite. Hence $\operatorname{Supp}\left(\sigma, \tau_{0}\right)$ is finite.

Remark 3.1.4. Note that if, for every $\sigma, \tau$ in $E$ and $a$ in $I, \min _{a}(I \backslash\{a \in I \mid r(a, \sigma, \tau)\})$ exists, then the same holds for $F$.

Lemma 3.1.5. Let $(E,\{r(a, \cdot, \cdot) \mid a \in I\})$ be a set together with a family of equivalence relations, and $\sigma \in E$. Set $\bar{\sigma}:=\left\{\sigma^{\prime} \mid \sigma^{\prime} \sim \sigma\right\}$, and $\kappa:=\max \left(\aleph_{0}, \operatorname{card}(I)\right)$. Assume the following.

- The $r(a, \cdot, \cdot)$ are non-trivial relations.

- $\operatorname{card}(\bar{\sigma})$ or $\operatorname{card}(I)$ is infinite.

- $(E,\{r(a, \cdot, \cdot) \mid a \in I\})$ is rich and $\kappa^{+}$-saturated.

Then $\operatorname{card}(\bar{\sigma})>\kappa$.

Proof. Assume that $\operatorname{card}(\bar{\sigma}) \leq \kappa$. We prove that the type $\{r(a, \tau, \sigma) \mid a \in I\} \cup\left\{\tau \neq \sigma^{\prime} \mid \sigma^{\prime} \in \bar{\sigma}\right\}$ is finitely realized. This holds if $\operatorname{card}(I)$ is finite, $\operatorname{since} \operatorname{card}(\bar{\sigma})$ is infinite. Assume that $\operatorname{card}(I)$ is infinite. A finite subset of this type is a set

$$
\left\{r\left(a_{1}, \tau, \sigma\right), \ldots, r\left(a_{n}, \tau, \sigma\right), \tau \neq \sigma_{1}, \ldots, \tau \neq \sigma_{p}\right\} .
$$

We take $a_{n+1}, \ldots, a_{n+p}$ in $I$ such that

$$
\operatorname{card}\left(\left\{a_{1}, \ldots, a_{n}, a_{n+1}, \ldots, a_{n+p}\right\}\right)=n+p .
$$

Since $E$ is rich, it contains some $\tau$ such that

$$
r\left(a_{1}, \tau, \sigma\right), \ldots, r\left(a_{n}, \tau, \sigma\right), \neg r\left(a_{n+1}, \tau, \sigma\right), \ldots, \neg r\left(a_{n+p}, \tau, \sigma\right) .
$$

Then $\tau$ satisfies $r\left(a_{1}, \tau, \sigma\right), \ldots, r\left(a_{n}, \tau, \sigma\right), \tau \neq \sigma_{1}, \ldots, \tau \neq \sigma_{p}$. By saturation, the type is realized, a contradiction which proves our lemma.

Note that if $I$ is finite, then $\sigma \sim \tau \Leftrightarrow \bigwedge_{a \in I} r(a, \sigma, \tau)$. So " $\sigma \sim \tau$ " is a first-order formula.

Theorem 3.1.6. Let $(E,\{r(a, \cdot, \cdot) \mid a \in I\})$ and $\left(E^{\prime},\left\{r^{\prime}(a, \cdot, \cdot) \mid a \in I\right\}\right)$ be two sets together with families of non-trivial equivalence relations. Assume the following.

- $(E,\{r(a, \cdot, \cdot) \mid a \in I\})$ and $\left(E^{\prime},\left\{r^{\prime}(a, \cdot, \cdot) \mid a \in I\right\}\right)$ are rich.

- For every $a \in I$, either $E / r(a, \cdot, \cdot)$ and $E^{\prime} / r^{\prime}(a, \cdot, \cdot)$ are infinite or $\operatorname{card} E / r(a, \cdot, \cdot)=\operatorname{card} E^{\prime} / r^{\prime}(a, \cdot, \cdot)$.

- If $\operatorname{card}(I)<\infty$, then either for every $\sigma \in E, \sigma^{\prime} \in E^{\prime}, \operatorname{card}(\{\tau \in E \mid \tau \sim \sigma\})$ and $\operatorname{card}\left(\left\{\tau^{\prime} \in E^{\prime} \mid \tau^{\prime} \sim\right.\right.$ $\left.\left.\sigma^{\prime}\right\}\right)$ are infinite or $\operatorname{card}(\{\tau \in E \mid \tau \sim \sigma\})=\operatorname{card}\left(\left\{\tau^{\prime} \in E^{\prime} \mid \tau^{\prime} \sim \sigma^{\prime}\right\}\right)$.

Then $(E,\{r(a, \cdot, \cdot) \mid a \in I\}) \equiv\left(E^{\prime},\left\{r^{\prime}(a, \cdot, \cdot) \mid a \in I\right\}\right)$ in $\mathcal{L} r e l_{I}$.

Proof. Let $\kappa=\max \left(\aleph_{0}, \operatorname{card}(I)\right)$. Without loss of generality, we can assume that both of $(E,\{r(a, \cdot, \cdot) \mid$ $a \in I\})$ and $\left(E^{\prime},\left\{r^{\prime}(a, \cdot, \cdot) \mid a \in I\right\}\right)$ are $\kappa^{+}$-saturated. We construct a family of isomorphisms between substructures of $E$ and $E^{\prime}$ of cardinal lower than $\kappa^{+}$, having the back-and-forth property.

We take $\sigma_{0}$ in $E, \sigma_{0}^{\prime}$ in $E^{\prime}$, and we set $f_{0}\left(\sigma_{0}\right):=\sigma_{0}^{\prime}$. Assume that $f_{1}: E_{1} \rightarrow E_{1}^{\prime}$ is an isomorphism, with $\operatorname{card}\left(E_{1}\right) \leq \kappa$, and let $\sigma \in E \backslash E_{1}$. We express $I$ as the union $I:=I_{\mathrm{y}} \cup I_{\mathrm{n}}$, where:

$\forall a \in I_{\mathrm{y}}, \exists \sigma_{a} \in E_{1}, r\left(a, \sigma, \sigma_{a}\right)$ and

$\forall a \in I_{\mathrm{n}}, \forall \tau \in E_{1}, \neg r(a, \sigma, \tau)$.

Hence, the type of $\sigma$ over $E_{1}$ consist of $\bigcup_{a \in I_{\mathrm{y}}} r\left(a, \sigma, \sigma_{a}\right)$ and of $\bigcup_{a \in I_{\mathrm{n}}} \bigcup_{\tau \in E_{1}} \neg r(a, \sigma, \tau)$.

If $\sigma \sim \tau$ for some $\tau \in E_{1}$, then we let $f_{1}(\sigma)$ be any element of the equivalence class of $f_{1}(\tau)$ which is not in $f_{1}\left(E_{1}\right)$. This element exists, because either the cardinal of the equivalence class of $f_{1}(\tau)$ is greater than $\kappa$ or the cardinal of the class of $\varphi_{1}(\tau)$ is equal to the cardinal of the class of $\tau$.

Assume that for every $\tau$ in $E_{1}$, there exists $a \in I$ such that $\neg r(a, \sigma, \tau)$. By assumption, $\operatorname{card}(E / r(a, \cdot, \cdot))=$ $\operatorname{card}\left(E^{\prime} / r^{\prime}(a, \cdot, \cdot)\right)$, whenever one of these is finite. If both are infinite, then, by $\kappa^{+}$-saturation, they are greater than $\kappa$. It follows that, for every $a$ in $I_{\mathrm{n}}$ and $\tau_{1}, \ldots, \tau_{p}$ in $E_{1}$, there exists $\sigma^{\prime}$ in $E^{\prime}$ such that $\neg r^{\prime}\left(a, \sigma^{\prime}, f_{1}\left(\tau_{1}\right)\right), \ldots, \neg r^{\prime}\left(a, \sigma^{\prime}, f_{1}\left(\tau_{p}\right)\right)$. Since $E^{\prime}$ is rich, every finite subset of the type

$$
\bigcup_{a \in I_{\mathrm{y}}} r^{\prime}\left(a, \sigma^{\prime}, f_{1}\left(\sigma_{a}\right)\right) \wedge \bigcup_{a \in I_{\mathrm{n}}} \bigcup_{\tau \in E_{1}} \neg r^{\prime}\left(a, \sigma^{\prime}, f_{1}(\tau)\right)
$$

is realized in $E^{\prime}$. By $\kappa^{+}$-saturation there exists $\sigma^{\prime}$ in $E^{\prime}$ such that $\bigcup_{a \in I_{y}} r^{\prime}\left(a, \sigma^{\prime}, f_{1}\left(\sigma_{a}\right)\right) \wedge \bigcup_{a \in I_{\mathrm{n}}} \bigcup_{\tau \in E_{1}} \neg r^{\prime}\left(a, \sigma^{\prime}, f_{1}((\tau))\right.$. 
3.2. Existentially equivalent cyclically ultrametric spaces. Cyclically ultrametric spaces will be structures of the language $\mathcal{L}_{C, r, t}=(C, r(\cdot, \cdot, \cdot),(\cdot, \cdot, \cdot))$, where $C$ a one place predicate. The concept of a cyclically ultrametric spaces $(E, u)$ such that $u$ is support-definable may be defined by the following system of axioms.

$\forall a, \forall b, \forall c,(a, b, c) \Rightarrow C(a) \& C(b) \& C(c) \& a \neq b \neq c \neq a \&(b, c, a) \& \neg(a, c, b)$

$\forall a, \forall b, \forall c, C(a) \& C(b) \& C(c) \& a \neq b \neq c \neq a \Rightarrow(a, b, c)$ or $(a, c, b)$

$\forall a, \forall b, \forall c, \forall d,(a, b, d) \&(a, d, c) \Rightarrow(a, b, c)$

$\forall a, \forall \sigma, C(a) \& \neg C(\sigma) \Rightarrow r(a, \sigma, \sigma)$

$\forall a, \forall \sigma, \forall \tau, r(a, \sigma, \tau) \Rightarrow C(a) \& \neg C(\sigma) \& \neg C(\tau) \& r(a, \tau, \sigma)$

$\forall a, \forall \sigma, \forall \tau, \forall \rho, r(a, \sigma, \rho) \& r(a, \rho, \tau) \Rightarrow r(a, \sigma, \tau)$

$\forall \sigma, \forall \tau, \neg C(\sigma) \& \neg C(\tau) \& \sigma \neq \tau \Rightarrow$

$[\forall a, C(a) \Rightarrow[\neg(r(a, \sigma, \tau)$ or $[\exists b, \neg r(b, \sigma, \tau) \& \forall c,(a, c, b) \Rightarrow r(c, \sigma, \tau)]]]$.

Furthermore, we will assume that the cyclically ultrametric spaces enjoy the following axiom, which states that the $r(a, \cdot, \cdot)$ are non-trivial relations:

$\forall a, C(a) \Rightarrow \exists \sigma, \exists \tau, \neg r(a, \sigma, \tau)$.

Definition 3.2.1. Let $(E, u, C)$ be a cyclically ultrametric space. We will say that $(E, u, C)$ is rich if $u$ is support-definable and the family $(r(a, \cdot, \cdot), a \in C)$ defined at the beginning of Subsection 3.1 is rich.

Remark 3.2.2. By Remark 3.1.4, every cyclically ultrametric space admits a rich closure, which can be constructed in same way as in Proposition 3.1.3.

Now, $(E, u, C)$ is rich if $u$ is support-definable and if it enjoys the following formulas:

$$
\begin{gathered}
\forall a_{1}, \ldots, \forall a_{n}, \forall a_{n+1}, \ldots, \forall a_{n+p}, \forall \sigma_{1}, \ldots, \forall \sigma_{n}, \forall \tau_{1,1}, \ldots, \forall \tau_{1, i_{1}}, \ldots, \forall \tau_{p, 1}, \ldots, \forall \tau_{p, i_{p}}, \\
C\left(a_{1}\right) \& \ldots \& C\left(a_{n}\right) \& C\left(a_{n+1}\right) \& \ldots \& C\left(a_{n+p}\right) \& \bigwedge_{i \neq j} a_{i} \neq a_{j} \\
\& \neg C\left(\sigma_{1}\right) \& \ldots \& \neg C\left(\sigma_{n}\right) \& \neg C\left(\tau_{1,1}\right) \& \ldots \& \neg C\left(\tau_{1, i_{1}}\right) \& \ldots \& \neg\left(\tau_{p, 1}\right) \& \ldots \\
\& \neg C\left(\tau_{p, i_{p}}\right) \& \exists \tau_{1}, \ldots, \exists \tau_{p}, \neg C\left(\tau_{1}\right) \& \ldots \& \neg C\left(\tau_{p}\right) \& \\
\neg r\left(a_{n+1}, \tau_{1}, \tau_{1,1}\right), \ldots, \neg r\left(a_{n+1}, \tau_{1}, \tau_{1, i_{1}}\right), \ldots, \neg r\left(a_{n+p}, \tau_{p}, \tau_{p, 1}\right), \ldots, \neg r\left(a_{n+p}, \tau_{p}, \tau_{p, i_{p}}\right) \Rightarrow \\
\exists \sigma, r\left(a_{1}, \sigma_{1}, \sigma\right), \ldots, r\left(a_{n}, \sigma_{n}, \sigma\right), \neg r\left(a_{n+1}, \sigma, \tau_{1,1}\right), \ldots, \neg r\left(a_{n+1}, \sigma, \tau_{1, i_{1}}\right), \ldots, \\
\neg r\left(a_{n+p}, \sigma, \tau_{p, 1}\right), \ldots, \neg r\left(a_{n+p}, \sigma, \tau_{p, i_{p}}\right),
\end{gathered}
$$

where $(n, p)$ run over all pairs of positive integers, and $\left(i_{1}, \ldots, i_{p}\right)$ run over all $p$-uples of positive integers.

Theorem 3.2.3. Let $(E, u, C)$ and $\left(E^{\prime}, u^{\prime}, C\right)$ be two rich cyclically ultrametric spaces. Assume that for every $a \in I$, either $E / r(a, \cdot, \cdot)$ and $E^{\prime} / r^{\prime}(a, \cdot, \cdot)$ are infinite or card $E / r(a, \cdot, \cdot)=$ card $E^{\prime} / r^{\prime}(a, \cdot, \cdot)$. Let $n, p$ be two integers, for $1 \leq i \leq n+p$, let $Q_{i} \in\{\forall, \exists\}$, and let $\varphi\left(a_{1}, \ldots, a_{n}, \sigma_{1}, \ldots, \sigma_{p}\right)$ be $a$ quantifier-free formula for the language $((\cdot, \cdot, \cdot), r(\cdot, \cdot, \cdot))$. Then:

$$
\begin{gathered}
(E, r, C) \models Q_{1} a_{1}, \ldots, Q_{n} a_{n}, Q_{n+1} \sigma_{1}, \ldots, Q_{n+p} \sigma_{p}, \bigwedge_{1 \leq i \leq n} C\left(a_{i}\right) \\
\& \bigwedge_{1 \leq i \leq p} \neg C\left(\sigma_{i}\right) \& \varphi\left(a_{1}, \ldots, a_{n}, \sigma_{1}, \ldots, \sigma_{p}\right)
\end{gathered}
$$

if and only if

$$
\begin{gathered}
\left(E^{\prime}, r^{\prime}, C\right) \models Q_{1} a_{1}, \ldots, Q_{n} a_{n}, Q_{n+1} \sigma_{1}, \ldots, Q_{n+p} \sigma_{p}, \bigwedge_{1 \leq i \leq n} C\left(a_{i}\right) \\
\& \bigwedge_{1 \leq i \leq p} \neg C\left(\sigma_{i}\right) \& \varphi\left(a_{1}, \ldots, a_{n}, \sigma_{1}, \ldots, \sigma_{p}\right) .
\end{gathered}
$$

In particular, $(E, r, C) \equiv_{\exists}\left(E^{\prime}, r^{\prime}, C\right)$ in $\mathcal{L}_{C, r, t}$.

Proof. Left to the reader (it is similar to the proof of Theorem 3.5.3).

Note that if we take $\equiv$ instead of $\equiv_{\exists}$, this result doesn't hold. For example, assume that $C$ is both infinite and well-ordered. For every $a \in C$, let $\Gamma_{a}$ be a non-trivial set. Fix some $O$ in $\prod_{a \in C} \Gamma_{a}$, and set $E:=\left\{\sigma \in \prod_{a \in C} \Gamma_{a} \mid \operatorname{card}(\operatorname{Supp}(\sigma, O)<\infty\}\right.$. Then $\prod_{a \in C} \Gamma_{a}$ enjoys $\forall \sigma, \exists \tau, \forall a, C(a) \Rightarrow \neg r(a, \sigma, \tau)$, but $E$ doesn't. 
Theorem 3. Let $(E, u, C)$ and $\left(E^{\prime}, u^{\prime}, C^{\prime}\right)$ be two rich cyclically ultrametric spaces such that $C \equiv C^{\prime}$. Assume that there exists $\kappa$ in $\{1,2,3, \ldots\} \cup\{\infty\}$ such that for every a in $C$ and for every $a^{\prime}$ in $C^{\prime}$, card $E / r(a, \cdot, \cdot)=$ card $E^{\prime} / r^{\prime}(a, \cdot, \cdot)=\kappa$. Let $n, p$ be two integers, for $1 \leq i \leq n+p$, let $Q_{i} \in\{\forall, \exists\}$, and let $\varphi\left(a_{1}, \ldots, a_{n}, \sigma_{1}, \ldots, \sigma_{p}\right)$ be a quantifier-free formula for the language $((\cdot, \cdot, \cdot), r(\cdot, \cdot, \cdot))$. Then:

$$
\begin{gathered}
(E, r, C) \models Q_{1} a_{1}, \ldots, Q_{n} a_{n}, Q_{n+1} \sigma_{1}, \ldots, Q_{n+p} \sigma_{p}, \bigwedge_{1 \leq i \leq n} C\left(a_{i}\right) \\
\& \bigwedge_{1 \leq i \leq p} \neg C\left(\sigma_{i}\right) \& \varphi\left(a_{1}, \ldots, a_{n}, \sigma_{1}, \ldots, \sigma_{p}\right)
\end{gathered}
$$

if and only if

$$
\begin{gathered}
\left(E^{\prime}, r^{\prime}, C^{\prime}\right) \models Q_{1} a_{1}, \ldots, Q_{n} a_{n}, Q_{n+1} \sigma_{1}, \ldots, Q_{n+p} \sigma_{p}, \\
\bigwedge_{1 \leq i \leq n} C\left(a_{i}\right) \& \bigwedge_{1 \leq i \leq p} \neg C\left(\sigma_{i}\right) \& \varphi\left(a_{1}, \ldots, a_{n}, \sigma_{1}, \ldots, \sigma_{p}\right) .
\end{gathered}
$$

In particular, $(E, r, C) \equiv_{\exists}\left(E^{\prime}, r^{\prime}, C^{\prime}\right)$ in $\mathcal{L}_{C, r, t}$.

Proof. If $C$ is finite, then $C^{\prime}$ is finite and $C^{\prime} \simeq C$. Hence the theorem follows from Theorem 3.2.3. Now, assume that both of $C$ and $C^{\prime}$ are infinite sets. Let $\alpha$ be a cardinal such that $\alpha>\max \left(\operatorname{card}(E), \operatorname{card}\left(E^{\prime}\right)\right)$ and $\alpha=\bigcup_{\beta<\alpha} 2^{\beta}$. We let $E_{1}$ (resp. $E_{1}^{\prime}$ ) be a special model of $E$ (resp. $E^{\prime}$ ) of cardinal $\alpha$ constructed in the same way as in [C-K 73] Proposition 5.1.8. Then $C_{1}, C_{1}^{\prime}$ are elementary equivalent special models of cardinal $\alpha$. Hence $C_{1} \simeq C_{1}^{\prime}$. Now, Theorem 3.2.3 serves to conclude the proof.

3.3. Generalized ultrametric spaces. The definition of generalized ultrametric spaces was given in [P-R 96]. A generalized ultrametric space is a set $E$ together with a mapping $d$ from $E \times E$ to an ordered set together with a greatest element $\infty$ such that:

$\forall \sigma \in E, \forall \tau \in E, d(\sigma, \tau)=d(\tau, \sigma)$

$\forall \sigma \in E, \forall \tau \in E, d(\sigma, \tau)=\infty \Leftrightarrow \sigma=\tau$

$\forall \sigma \in E, \forall \tau \in E, \forall \rho \in E, \forall a \in d(E \times E),(d(\sigma, \rho) \geq a \& d(\rho, \tau) \geq a) \Rightarrow d(\sigma, \tau) \geq a$.

The mapping $d$ is called a generalized ultrametric distance.

Let $C:=d(E \times E)$. For every $a \in C, \sigma \in E, \rho \in E$, we set $r_{d}(a, \sigma, \rho) \Leftrightarrow a \leq d(\sigma, \rho)$. Then $\left\{r_{d}(a, \cdot, \cdot) \mid a \in C\right\}$ is a family of equivalence relations. Indeed, let $a$ be an element of $C, \sigma, \rho, \tau$ be elements of $E$. $d(\sigma, \sigma)=\infty$, hence $r_{d}(a, \sigma, \sigma)$ holds. We have $r_{d}(a, \sigma, \rho) \Leftrightarrow r_{d}(a, \rho, \sigma)$, since $d(\sigma, \rho)=d(\rho, \sigma)$. Now, if $r_{d}(a, \sigma, \rho)$ and $r_{d}(a, \rho, \tau)$, then $a \leq d(\sigma, \rho)$ and $a \leq d(\rho, \tau)$. It follows: $a \leq d(\sigma, \tau)$, hence $r_{d}(a, \sigma, \tau)$. Furthermore, this family satisfies:

$$
\forall \sigma \in E, \forall \rho \in E, \sigma \neq \rho \Rightarrow \exists a \in C, \neg r_{d}(a, \sigma, \rho) .
$$

Conversely, assume that $E$ is a set together with a family of equivalence relations $\{r(a, \cdot, \cdot) \mid a \in C\}$ such that:

$$
\forall \sigma \in E, \forall \rho \in E, \sigma \neq \rho \Rightarrow \exists a \in C, \neg r(a, \sigma, \rho) .
$$

The relation $\forall a \in C,\left(r\left(a, \sigma_{1}, \rho_{1}\right) \Leftrightarrow r\left(a, \sigma_{2}, \rho_{2}\right)\right)$ is an equivalence relation on $E \times E$. We let $d_{r}\left(\sigma_{1}, \rho_{1}\right)$ be the class of $\left(\sigma_{1}, \rho_{1}\right)$. For every $\sigma_{1}, \rho_{1}, \sigma_{2}, \rho_{2}$ in $E$, set $d_{r}\left(\sigma_{1}, \rho_{1}\right) \leq d_{r}\left(\sigma_{2}, \rho_{2}\right) \Leftrightarrow \forall a \in C,\left(r\left(a, \sigma_{1}, \rho_{1}\right) \Rightarrow\right.$ $\left.r\left(a, \sigma_{2}, \rho_{2}\right)\right)$. One can check that the set $\left\{d_{r}(\sigma, \rho) \mid \sigma \in E, \rho \in E\right\}$ is an ordered set with greatest element $d_{r}(\sigma, \sigma)$ (for any $\sigma$ in $E$ ), and that $d_{r}$ is a generalized ultrametric distance.

If $(E, d)$ is a generalized ultrametric space, then it is isomorphic to $\left(E, d_{r_{d}}\right)$. Now, if $(E, r)$ is a set together with a family of equivalence relations, then $\left(E, r_{d_{r}}\right)$ needs not be isomorphic to $(E, r)$. Indeed, let $\sigma_{1}, \rho_{1}$ in $E$ and $d_{1}=d\left(\sigma_{1}, \rho_{1}\right)$. For any $\sigma, \rho$ in $E$, we have: $r_{d_{r}}\left(d_{1}, \sigma, \rho\right) \Leftrightarrow d_{1} \leq d_{r}(\sigma, \rho) \Leftrightarrow$ $\forall a \in C,\left(r\left(a, \sigma_{1}, \rho_{1}\right) \Rightarrow r(a, \sigma, \rho)\right) \Leftrightarrow \operatorname{Supp}(\sigma, \rho) \subset \operatorname{Supp}\left(\sigma_{1}, \rho_{1}\right)$. Now, if $\operatorname{Supp}\left(\sigma_{1}, \rho_{1}\right)=C \backslash\{a\}$, then $r_{d_{r}}\left(d_{1}, \cdot, \cdot\right)$ is $r\left(d_{1}, \cdot, \cdot\right)$. Otherwise, as an example, let $C:=\{a, b, c\}, E:=\left\{\sigma_{1}, \sigma_{2}, \sigma_{3}, \sigma_{4}\right\}, r(a, \cdot, \cdot)$ : $\left\{\left\{\sigma_{1}, \sigma_{3}, \sigma_{4}\right\},\left\{\sigma_{2}\right\}\right\}, r(b, \cdot, \cdot):\left\{\left\{\sigma_{1}, \sigma_{2}\right\},\left\{\sigma_{3}, \sigma_{4}\right\}\right\}$, and $r(c, \cdot, \cdot):\left\{\left\{\sigma_{1}, \sigma_{2}, \sigma_{3}\right\},\left\{\sigma_{4}\right\}\right\}$. Then $r_{d_{r}}$ defines the following equivalence relations:

$$
\begin{gathered}
\left\{\left\{\sigma_{1}\right\},\left\{\sigma_{2}\right\},\left\{\sigma_{3}\right\},\left\{\sigma_{4}\right\}\right\},\left\{\left\{\sigma_{1}, \sigma_{2}\right\},\left\{\sigma_{3}\right\},\left\{\sigma_{4}\right\}\right\},\left\{\left\{\sigma_{1}, \sigma_{3}\right\},\left\{\sigma_{2}\right\},\left\{\sigma_{4}\right\}\right\}, \\
\left\{\left\{\sigma_{1}\right\},\left\{\sigma_{2}\right\},\left\{\sigma_{3}, \sigma_{4}\right\}\right\},\left\{\left\{\sigma_{1}, \sigma_{2}, \sigma_{3}\right\},\left\{\sigma_{4}\right\}\right\}, \text { and }\left\{\left\{\sigma_{1}, \sigma_{3}, \sigma_{4}\right\},\left\{\sigma_{2}\right\}\right\} .
\end{gathered}
$$


Remark 3.3.1. Let $(E, d)$ be a generalized ultrametric space. We can equip $C=d(E \times E)$ with a cyclic well-order. Hence the range of $d_{r_{d}}$ is a subset of $\mathcal{L B}$ (defined in $\S 2.1$ ). Therefore, $d_{r_{d}}$ can be identified with the support mapping of a cyclically ultrametric space such that $u$ is support-definable. Thus, $E$ embeds in a cartesian product, and $d_{r_{d}}$ is its support mapping. Since $(E, d)$ is isomorphic to $\left(E, d_{r_{d}}\right)$, it embeds in the same cartesian product.

We will say that the generalized ultrametric space $(E, d)$ is rich if the family $\left(E, r_{d}\right)$ is rich.

Generalized ultrametric spaces, will be structures for the language $\mathcal{L}_{C, r}=(C, r(\cdot, \cdot, \cdot))$. The axioms for the theory of generalized ultrametric spaces can be listed as follows.

$\forall x, \forall y, \forall z, r(x, y, z) \Rightarrow C(x) \& \neg C(y) \& \neg C(z) \& r(x, z, y)$

$\forall x, \forall y, C(x) \& \neg C(y) \Rightarrow r(x, y, y)$

$\forall x, \forall y, \forall z, \forall t, r(x, y, t) \& r(x, t, z) \Rightarrow r(x, y, z)$

$\forall y, \forall z, \neg C(y) \& \neg C(z) \& y \neq z \Rightarrow \exists x, \neg r(x, y, z)$.

The family $\{r(a, \cdot, \cdot) \mid a \in C\}$ is rich if $E$ satisfies the formulas:

$$
\begin{gathered}
\forall x_{1}, \ldots, \forall x_{n}, \forall x_{n+1}, \ldots, \forall x_{n+p}, \forall y_{1}, \ldots, \forall y_{n}, \forall z_{1,1}, \ldots, \forall z_{1, i_{1}}, \ldots, \forall z_{p, 1}, \ldots, \forall z_{p, i_{p}}, \\
C\left(x_{1}\right) \& \ldots \& C\left(x_{n}\right) \& C\left(x_{n+1}\right) \& \ldots \& C\left(x_{n+p}\right) \& \bigwedge_{i \neq j} x_{i} \neq x_{j} \\
\& \neg C\left(y_{1}\right) \& \ldots \& \neg C\left(y_{n}\right) \\
\& \neg C\left(z_{1,1}\right) \& \ldots \& \neg C\left(z_{1, i_{1}}\right) \ldots \& \neg C\left(z_{p, 1}\right) \& \ldots \& \neg C\left(z_{p, i_{p}}\right) \\
\& \exists z_{1}, \ldots, \exists z_{p}, \neg C\left(z_{1}\right) \& \ldots \& \neg C\left(z_{p}\right) \\
\& \neg r\left(x_{n+1}, z_{1}, z_{1,1}\right), \ldots, \neg r\left(x_{n+1}, z_{1}, z_{1, i_{1}}\right), \ldots, \\
\neg r\left(x_{n+p}, z_{p}, z_{p, 1}\right), \ldots, \neg r\left(x_{n+p}, z_{p}, z_{p, i_{p}}\right) \Rightarrow \\
\exists y, r\left(x_{1}, y_{1}, y\right), \ldots, r\left(x_{n}, y_{n}, y\right), \neg r\left(x_{n+1}, y, z_{1,1}\right), \ldots, \neg r\left(x_{n+1}, y, z_{1, i_{1}}\right), \ldots, \\
\neg r\left(x_{n+p}, y, z_{p, 1}\right), \ldots, \neg r\left(x_{n+p}, y, z_{p, i_{p}}\right),
\end{gathered}
$$

where $(n, p)$ run over all pairs of positive integers, and $\left(i_{1}, \ldots, i_{p}\right)$ run over all $p$-uples of positive integers.

The following theorems can be proved in the same way as Theorem 3.2.3 and Theorem 3 respectively.

Theorem 3.3.2. Let $(E, C, r)$ and $\left(E^{\prime}, C^{\prime}, r^{\prime}\right)$ be two rich generalized ultrametric spaces such that the families $\{r(a, \cdot, \cdot) \mid a \in C\}$ and $\left\{r^{\prime}\left(a^{\prime}, \cdot, \cdot\right) \mid a^{\prime} \in C^{\prime}\right\}$ are rich. We assume that there exists a bijection $f$ from $C$ onto $C^{\prime}$ such that for every $a \in C$, either both of $E / r(a, \cdot, \cdot)$ and $E^{\prime} / r^{\prime}(f(a), \cdot, \cdot)$ are infinite, or card $(E / r(a, \cdot, \cdot))=\operatorname{card}\left(E^{\prime} / r^{\prime}(f(a), \cdot, \cdot)\right)$. Let $n, p$ be two integers, for $1 \leq i \leq n+p$, let $Q_{i} \in\{\forall, \exists\}$, and let $\varphi\left(x_{1}, \ldots, x_{n}, y_{1}, \ldots, y_{p}\right)$ be a quantifier-free formula for the language $r(\cdot, \cdot, \cdot)$. Then:

$$
\begin{gathered}
(E, C, r) \models Q_{1} x_{1}, \ldots, Q_{n} x_{n}, Q_{n+1} y_{1}, \ldots, Q_{n+p} y_{p}, \bigwedge_{1 \leq i \leq n} C\left(x_{i}\right) \& \bigwedge_{1 \leq i \leq p} \neg C\left(y_{i}\right) \\
\& \varphi\left(x_{1}, \ldots, x_{n}, y_{1}, \ldots, y_{p}\right)
\end{gathered}
$$

if and only if

$$
\begin{gathered}
\left(E^{\prime}, C^{\prime}, r^{\prime}\right) \models Q_{1} x_{1}, \ldots, Q_{n} x_{n}, Q_{n+1} y_{1}, \ldots, Q_{n+p} y_{p}, \bigwedge_{1 \leq i \leq n} C\left(x_{i}\right) \& \bigwedge_{1 \leq i \leq p} \neg C\left(y_{i}\right) \\
\& \varphi\left(x_{1}, \ldots, x_{n}, y_{1}, \ldots, y_{p}\right) .
\end{gathered}
$$

In particular, $(E, C, r) \equiv_{\exists}\left(E^{\prime}, C^{\prime}, r^{\prime}\right)$ in $\mathcal{L}_{r, C}$.

Theorem 3.3.3. Let $(E, C, r)$ and $\left(E^{\prime}, C^{\prime}, r^{\prime}\right)$ be two rich generalized ultrametric spaces such that both of the families $\{r(a, \cdot, \cdot) \mid a \in C\}$ and $\left\{r^{\prime}\left(a^{\prime}, \cdot, \cdot\right) \mid a^{\prime} \in C^{\prime}\right\}$ are rich. We assume that either both of $C$ and $C^{\prime}$ are infinite or card $(C)=$ card $\left(C^{\prime}\right)$, and that there exists $\kappa \in\{1,2,3, \ldots\} \cup\{\infty\}$ such that for every $a \in C$ and $a^{\prime} \in C^{\prime}$, card $(E / r(a, \cdot, \cdot))=\operatorname{card}\left(E^{\prime} / r^{\prime}\left(a^{\prime}, \cdot \cdot \cdot\right)\right)=\kappa$. Let $n, p$ be two integers, for $1 \leq i \leq n+p$, let $Q_{i} \in\{\forall, \exists\}$, and let $\varphi\left(x_{1}, \ldots, x_{n}, y_{1}, \ldots, y_{p}\right)$ be a quantifier-free formula for the language $r(\cdot, \cdot, \cdot)$. Then:

$$
\begin{gathered}
(E, C, r) \models Q_{1} x_{1}, \ldots, Q_{n} x_{n}, Q_{n+1} y_{1}, \ldots, Q_{n+p} y_{p}, \bigwedge_{1 \leq i \leq n} C\left(x_{i}\right) \& \bigwedge_{1 \leq i \leq p} \neg C\left(y_{i}\right) \\
\& \varphi\left(x_{1}, \ldots, x_{n}, y_{1}, \ldots, y_{p}\right)
\end{gathered}
$$


if and only if

$$
\begin{gathered}
\left(E^{\prime}, C, r^{\prime}\right) \models Q_{1} x_{1}, \ldots, Q_{n} x_{n}, Q_{n+1} y_{1}, \ldots, Q_{n+p} y_{p}, \bigwedge_{1 \leq i \leq n} C\left(x_{i}\right) \& \bigwedge_{1 \leq i \leq p} \neg C\left(y_{i}\right) \\
\& \varphi\left(x_{1}, \ldots, x_{n}, y_{1}, \ldots, y_{p}\right) .
\end{gathered}
$$

In particular, $(E, C, r) \equiv_{\exists}\left(E^{\prime}, C, r^{\prime}\right)$ in $\mathcal{L}_{C, r}$.

3.4. Families of subgroups. Let $(G,+, v)$ be a cyclically valued group such that $v$ is defined by the supports. One easily checks that, for every $a \in C$, the set $\{\sigma \in G \mid v(a, \sigma) \neq a\}$ is a subgroup of $G$. Furthermore, $\forall \sigma \in G,(\forall a \in C, v(a, \sigma) \neq a) \Rightarrow \sigma=0$. Conversely, we have the following.

Lemma 3.4.1. Let $G$ be a non-trivial abelian group, and $G_{a}, a \in C$, be a family of subgroups of $G$. Assume that for every $a \in C$ and every $\sigma \neq 0$ in $E, \min _{a}\left\{a \in C \mid \sigma \notin G_{a}\right\}$ exists. We set $v(a, \sigma):=\min _{a}\left\{b \in C \mid \sigma \notin G_{b}\right\}$, and $v(a, 0):=\infty$. Then:

1) $(G,+, v)$ is a cyclically valued group such that $v$ is support-definable,

2) for every $a \in C$ and every $\sigma$ in $E, \sigma \in G_{a} \Leftrightarrow v(a, \sigma) \neq a$.

Proof. Let $a \in C$ and $\sigma, \tau$ in $G$. Since $\left(\sigma-\tau \notin G_{a}\right) \Rightarrow\left(\sigma \notin G_{a}\right.$ or $\left.\tau \notin G_{a}\right),\left\{a \in C \mid \sigma-\tau \notin G_{a}\right\} \subset$ $\left\{a \in C \mid \sigma \notin G_{a}\right\} \cup\left\{a \in C \mid \tau \notin G_{a}\right\}$. Hence $v(a, \sigma-\tau) \geq_{a} \min _{a}(v(a, \sigma), v(a, \tau))$. Therefore $(G,+, v)$ is a cyclically valued group.

As a consequence of hypothesis, it follows that $v(a, \sigma)=a \Leftrightarrow a \in\left\{b \in C \mid \sigma \notin G_{b}\right\}$. Hence $v(a, \sigma) \neq a \Leftrightarrow \sigma \in G_{a}$. Therefore $\operatorname{Supp}(\sigma)=\{a \in C \mid v(a, \sigma)=a\}=\left\{a \in C \mid \sigma \notin G_{a}\right\}$. Hence $v(a, \sigma)=\min _{a} \operatorname{Supp}(\sigma)$. So $v$ is support-definable.

Let $I$ be a set and $G$ be an abelian group. Assume that there exists a family $G_{a}, a \in I$, of non-trivial subgroups of $G$. For every $\sigma$ in $G$, the support of $\sigma$ will be the set $\operatorname{Supp}(\sigma):=\left\{a \in I \mid \sigma \notin G_{a}\right\}$. We set $G_{\mathrm{n}}:=\bigcap_{a \in C} G_{a}$.

For example, let $I$ be a non-empty set, and for every $a \in I$ let $\Gamma_{a}$ be a non-trivial abelian group. We let $G$ be the cartesian product $\prod_{a \in I} \Gamma_{a}$, and for every $b \in I, \sigma=\left(\sigma_{a}\right)_{a \in I}$ in $G$, we set $\sigma \in G_{b}$ if and only if $\sigma_{b}=0$. Obviously, for every $a \in I, G_{a}$ is a subgroup of $G$ and $G_{a}$ is isomorphic to $\left(\prod_{b \in I, b \neq a} \Gamma_{b}\right) \times\{0\}$.

Let $\left(G,\left\{G_{a} \mid a \in I\right\}\right)$ be an abelian group together with a family of subgroups. For every $a \in$ $I$, set $\Gamma_{a}:=G / G_{a}$. We see that there is a natural embedding from $\left(G / G_{\mathrm{n}},\left\{G_{a} / G_{\mathrm{n}} \mid a \in I\right\}\right)$ to $\left(\prod_{a \in I} \Gamma_{a},\left\{\left(\prod_{b \in I, b \neq a} \Gamma_{b}\right) \times\{0\} \mid a \in I\right\}\right)$.

Let $\left(G,\left\{G_{a} \mid a \in I\right\}\right)$ be an abelian group together with a family of subgroups, and $(a, g) \in I \times G$. Set $g(a, \sigma) \Leftrightarrow \sigma \in G_{a}$.

The language $\mathcal{L} g_{I}$ will be the language of groups together with unary symbols $g(a, \cdot)$, one for each $a \in I$. So $G_{\mathrm{n}}$ will be the subgroup $\{\sigma \in G \mid \forall a \in I, g(a, \sigma)\}$.

For every finite subset $\left\{a_{1}, \ldots, a_{n}, a_{n+1}, \ldots, a_{n+p}\right\}$ of $I$ and $p$-uple $\left\{i_{1}, \ldots, i_{p}\right\}$ of positive integers,

$$
\Phi_{a_{1}, \ldots, a_{n} ; a_{n+1}, \ldots, a_{n+p} ; i_{1}, \ldots, i_{p}}\left(\sigma_{1}, \ldots, \sigma_{n}, \tau_{1,1}, \ldots, \tau_{1, i_{1}}, \ldots, \tau_{p, 1}, \ldots, \tau_{p, i_{p}}\right)
$$

will be the formula

$$
\begin{gathered}
\exists \tau_{1}, \ldots, \exists \tau_{p}, \neg g\left(a_{n+1}, \tau_{1}-\tau_{1,1}\right), \ldots, \neg g\left(a_{n+1}, \tau_{1}-\tau_{1, i_{1}}\right), \ldots, \\
\neg g\left(a_{n+p}, \tau_{p}-\tau_{p, 1}\right), \ldots, \neg g\left(a_{n+p}, \tau_{p}-\tau_{p, i_{p}}\right) \Rightarrow \\
\exists \sigma, g\left(a_{1}, \sigma_{1}-\sigma\right), \ldots, g\left(a_{n}, \sigma_{n}-\sigma\right), \neg g\left(a_{n+1}, \sigma-\tau_{1,1}\right), \ldots, \neg g\left(a_{n+1}, \sigma-\tau_{1, i_{1}}\right), \ldots, \\
\neg g\left(a_{n+p}, \sigma-\tau_{p, 1}\right), \ldots, \neg g\left(a_{n+p}, \sigma-\tau_{p, i_{p}}\right) .
\end{gathered}
$$

Note that if all the subgroups $G_{a}, a \in I$, are infinite, then

$$
\Phi_{a_{1}, \ldots, a_{n} ; a_{n+1}, \ldots, a_{n+p} ; i_{1}, \ldots, i_{p}}\left(\sigma_{1}, \ldots, \sigma_{n}, \tau_{1,1}, \ldots, \tau_{1, i_{1}}, \ldots, \tau_{p, 1}, \ldots, \tau_{p, i_{p}}\right)
$$

is equivalent to:

$$
\begin{gathered}
\exists \sigma, g\left(a_{1}, \sigma_{1}-\sigma\right), \ldots, g\left(a_{n}, \sigma_{n}-\sigma\right), \neg g\left(a_{n+1}, \sigma-\tau_{1,1}\right), \ldots, \neg g\left(a_{n+1}, \sigma-\tau_{1, i_{1}}\right), \ldots, \\
\neg g\left(a_{n+p}, \sigma-\tau_{p, 1}\right), \ldots, \neg g\left(a_{n+p}, \sigma-\tau_{p, i_{p}}\right) .
\end{gathered}
$$


Definition 3.4.2. Let $\left(G,\left\{G_{a} \mid a \in I\right\}\right)$ be an abelian group together with a family of non-trivial subgroups. We will say that $\left(G,\left\{G_{a} \mid a \in I\right\}\right)$ is rich if and only if for every formula

$\Phi_{a_{1}, \ldots, a_{n} ; a_{n+1}, \ldots, a_{n+p} ; i_{1}, \ldots, i_{p}}$, where $\operatorname{card}\left(\left\{a_{1}, \ldots, a_{n}, a_{n+1}, \ldots, a_{n+p}\right\}=n+p\right.$,

$\left(G,\left\{G_{a} \mid a \in I\right\}\right)$ enjoys

$$
\begin{gathered}
\forall \sigma_{1}, \ldots, \forall \sigma_{n}, \forall \tau_{1,1}, \ldots, \forall \tau_{1, i_{1}}, \ldots, \forall \tau_{p, 1}, \ldots, \forall \tau_{p, i_{p}}, \\
\Phi_{a_{1}, \ldots, a_{n} ; a_{n+1}, \ldots, a_{n+p} ; i_{1}, \ldots, i_{p}}\left(\sigma_{1}, \ldots, \sigma_{n}, \tau_{1,1}, \ldots, \tau_{1, i_{1}}, \ldots, \tau_{p, 1}, \ldots, \tau_{p, i_{p}}\right) .
\end{gathered}
$$

Lemma 3.4.3. Let $\left(G,\left\{G_{a} \mid a \in I\right\}\right)$ be an abelian group together with a family of non-trivial subgroups. We have already mentioned that $G / G_{\mathrm{n}}$ embeds in a natural way in $\prod_{a \in I} \Gamma_{a}$, where $\Gamma_{a}=G / G_{a}$. If the image of $G / G_{\mathrm{n}}$ under this embedding contains $\sum_{a \in I} \Gamma_{a}$, then $(G,\{g(a, \cdot) \mid a \in I\})$ is rich.

Proof. Assume that $\sum_{a \in I} \Gamma_{a}$ is a subset of the image of $G / G_{\mathrm{n}}$. Let $a_{1}, \ldots, a_{n}, a_{n+1}, \ldots, a_{n+p}$ in $I$, $i_{1}, \ldots, i_{p}$ be positive integers, and $\sigma_{1}, \ldots, \sigma_{n}, \tau_{1,1}, \ldots, \tau_{1, i_{1}}, \ldots, \tau_{p, 1}, \ldots, \tau_{p, i_{p}}, \tau_{1}, \ldots, \tau_{p}$ in $G$ such that:

$$
\neg g\left(a_{n+1}, \tau_{1}-\tau_{1,1}\right), \ldots, \neg g\left(a_{n+1}, \tau_{1}-\tau_{1, i_{1}}\right), \ldots, \neg g\left(a_{n+p}, \tau_{p}-\tau_{p, 1}\right), \ldots, \neg g\left(a_{n+p}, \tau_{p}-\tau_{p, i_{p}}\right) .
$$

Denote by $\left(\sigma_{a}\right)_{a \in I}$ the image in $\prod_{a \in I} \Gamma_{a}$ of the class of any element $\sigma$ of $G$. For every $a \in I$, we have: $g(a, \sigma) \Leftrightarrow \sigma_{a}=0$. For $1 \leq i \leq n$, set $\sigma_{a_{i}}:=\sigma_{i a_{i}}$, for $1 \leq i \leq p$, set $\sigma_{a_{n+i}}:=\tau_{i a_{n+i}}$, otherwise, set $\sigma_{a}=0$. Let $\sigma$ in $G$ such that the image of the class of $\sigma$ is $\left(\sigma_{a}\right)_{a \in I}$. Then $\sigma$ enjoys $g\left(a_{1}, \sigma_{1}-\right.$ $\sigma), \ldots, g\left(a_{n}, \sigma_{n}-\sigma\right), \neg g\left(a_{n+1}, \sigma-\tau_{1,1}\right), \ldots, \neg g\left(a_{n+1}, \sigma-\tau_{1, i_{1}}\right), \ldots, \neg g\left(a_{n+p}, \sigma-\tau_{p, 1}\right), \ldots, \neg g\left(a_{n+p}, \sigma-\right.$ $\left.\tau_{p, i_{p}}\right)$. Hence, $(G,\{g(a, \cdot) \mid a \in I\})$ is rich.

Proposition 3.4.4. Let $\left(G,\left\{G_{a} \mid a \in I\right\}\right)$ be an abelian group together with a family of non-trivial subgroups. Assume that $G_{\mathrm{n}}=\{0\}$. Then, there exists an abelian group $H$, such that $G \subset H$, and for every $a \in I$, there exists an extension of $g(a, \cdot)$ to $H$ such that $(H,\{g(a, \cdot) \mid a \in I\})$ is rich. For every $\sigma \in H$, there exists $\tau \in G$ such that $\operatorname{Supp}(\sigma-\tau)$ is finite. Furthermore, $H \hookrightarrow \prod_{a \in I} \Gamma_{a}$.

Proof. $G / G_{\mathrm{n}} \simeq G$, hence $G$ embeds in a natural way in $\prod_{a \in I} \Gamma_{a}$. Therefore we assume that $G$ is a subgroup of $\prod_{a \in I} \Gamma_{a}$. We extend $g$ to $\prod_{a \in I} \Gamma_{a}$ as follows. Let $a_{0} \in I$ and $\sigma=\left(\sigma_{a}\right)_{a \in I}$ in $\prod_{a \in I} \Gamma_{a}$. We set $g\left(a_{0}, \sigma\right) \Leftrightarrow \sigma_{a_{0}}=0$.

Now, we set $H:=G+\sum_{a \in I} \Gamma_{a}$. By Lemma 3.4.3, $H$ is rich. For every $\sigma \in H$, there exists $\tau \in G$ such that $\operatorname{Supp}(\sigma-\tau)$ is finite. Trivially, $H \hookrightarrow \prod_{a \in I} \Gamma_{a}$.

Remark 3.4.5. Note that if, for every $\sigma$ in $G$ and $a$ in $I, \min _{a}\{a \in I \mid \neg g(a, \sigma)\}$ exists, then the same holds for $H$.

Lemma 3.4.6. Let $\left(G,\left\{G_{a} \mid a \in I\right\}\right)$ be an abelian group together with a family non-trivial subgroups. Assume that $(G,\{g(a, \cdot) \mid a \in I\})$ is rich and card $(I)^{+}$-saturated. Then:

a) the image of $G$ in $\prod_{a \in I} \Gamma_{a}$ contains $\sum_{a \in I} \Gamma_{a}$,

b) if $\operatorname{card}(I)$ is infinite, then $\operatorname{card}\left(G_{\mathrm{n}}\right)>\operatorname{card}(I)$.

Proof. .

a) Let $\left(\rho_{a}\right)_{a \in I}$ in $\sum_{a \in I} \Gamma_{a}$, and $\left\{a_{1}, \ldots, a_{n}\right\}$ be the support of $\left(\rho_{a}\right)_{a \in I}$. For $i \in\{1, \ldots, n\}$, we let $\sigma_{i} \in G$ be such that $\sigma_{i a_{i}}=\rho_{a_{i}}$. Now, the type $g\left(a_{1}, \sigma-\sigma_{1}\right), \ldots, g\left(a_{n}, \sigma-\sigma_{n}\right)$, and $g(a, 0)$, for $a \notin\left\{a_{1}, \ldots, a_{n}\right\}$ is finitely realized, since $G$ is rich. Hence it is realized by some $\sigma$ in $G$. Then the image of $\sigma$ is $\left(\rho_{a}\right)_{a \in I}$.

The proof of b) is similar to the proof of Lemma 3.1.5, and it is left to the reader.

Note that if $\operatorname{card}(I)<\infty$, then $G_{\mathrm{n}}=\{0\} \Leftrightarrow\left[\forall \sigma \in G,\left(\bigwedge_{a \in I} g(a, \sigma)\right) \Rightarrow \sigma=0\right]$. Hence $G_{\mathrm{n}}=\{0\}$ is a first-order sentence.

Theorem 3.4.7. Let $\left(G,\left\{G_{a} \mid a \in I\right\}\right)$ and $\left(G^{\prime},\left\{g^{\prime}(a, \cdot) \mid a \in I\right\}\right)$ be two torsion-free divisible abelian groups together with families of subgroups. Assume that:

both of $G$ and $G^{\prime}$ are rich,

if $\operatorname{card}(I)<\infty$, then $G_{\mathrm{n}}=\{0\} \Leftrightarrow G_{\mathrm{n}}^{\prime}=\{0\}$,

and for every $a \in I$ :

- both of $G_{a}=\{\sigma \in G \mid g(a, \sigma)\}$ and $G_{a}^{\prime}=\left\{\sigma^{\prime} \in G^{\prime} \mid g^{\prime}\left(a, \sigma^{\prime}\right)\right\}$ are divisible,

- $G_{a}=G \Leftrightarrow G_{a}^{\prime}=G^{\prime}$.

Then $(G,\{g(a, \cdot) \mid a \in I\}) \equiv\left(G^{\prime},\left\{g^{\prime}(a, \cdot) \mid a \in I\right\}\right)$ in $\mathcal{L} g_{I}$. 
Proof. Let $\kappa=\max \left(\aleph_{0}, \operatorname{card}(I)\right)$. Without loss of generality, we can assume that both of $(G,\{g(a, \cdot) \mid$ $a \in I\})$ and $\left(G^{\prime},\left\{g^{\prime}(a, \cdot) \mid a \in I\right\}\right)$ are $\kappa^{+}$-saturated. For every $a \in I$, we set $\Gamma_{a}:=G /\{\sigma \in G \mid g(a, \sigma)\}$ and $\Gamma_{a}^{\prime}:=G^{\prime} /\left\{\sigma^{\prime} \in G^{\prime} \mid g^{\prime}\left(a^{\prime}, \sigma^{\prime}\right)\right\}$. Note that both of $\Gamma_{a}, \Gamma_{a}^{\prime}, G_{\mathrm{n}}$ and $G_{\mathrm{n}}^{\prime}$ are divisible. Hence their cardinals are infinite, whenever they are non-trivial. Consequently, by $\kappa^{+}$-saturation, their cardinals are greater than $\kappa$.

The theorem will be proved by showing that there exists a family of isomorphisms between substructures of $G$ and $G^{\prime}$ of cardinal at most $\kappa$, which has the back-and-forth property.

There exists a natural isomorphism from $\{0\} \subset G$ onto $\{0\} \subset G^{\prime}$.

Assume that $f_{0}: G_{0} \rightarrow G_{0}^{\prime}$ is a partial isomorphism, with $\operatorname{card}\left(G_{0}\right) \leq \operatorname{card}(I)$ and $G_{0}$ divisible. Let $\sigma \in G \backslash G_{0}$, and $G_{1}$ be the divisible hull of the subgroup generated by $G_{0}+\sigma$. Let $I_{\mathrm{y}}:=\left\{a \in I \mid \exists \sigma_{a} \in G_{0}, g\left(a, \sigma-\sigma_{a}\right)\right\}$ and

$I_{\mathrm{n}}:=\left\{a \in I \mid \forall \tau \in G_{0}, \neg g(a, \sigma-\tau)\right\}$.

We exhibit an element $\sigma^{\prime}$ of $G^{\prime}$ such that:

$$
\sigma^{\prime} \notin G_{0}^{\prime}, \bigwedge_{a \in I_{\mathrm{y}}} g^{\prime}\left(a, \sigma^{\prime}-f_{0}\left(\sigma_{a}\right)\right) \text { and } \bigwedge_{a \in I_{\mathrm{n}}} \bigwedge_{\tau \in G_{0}} \neg g^{\prime}\left(a, \sigma^{\prime}-f_{0}(\tau)\right) .
$$

Let the formula formula:

$$
\begin{gathered}
g^{\prime}\left(a_{1}, \sigma^{\prime}-f_{0}\left(\sigma_{a_{1}}\right)\right), \ldots, g^{\prime}\left(a_{n}, \sigma^{\prime}-f_{0}\left(\sigma_{a_{n}}\right)\right) \\
\neg g^{\prime}\left(a_{n+1}, \sigma^{\prime}-f_{0}\left(\tau_{1,1}\right)\right), \ldots, \neg g^{\prime}\left(a_{n+1}, \sigma^{\prime}-f_{0}\left(\tau_{1, i_{1}}\right)\right), \ldots, \\
\neg g^{\prime}\left(a_{n+p}, \sigma^{\prime}-f_{0}\left(\tau_{p, 1}\right)\right), \ldots, \neg g^{\prime}\left(a_{n+p}, \sigma^{\prime}-f_{0}\left(\tau_{p, i_{p}}\right)\right),
\end{gathered}
$$

where $\sigma_{a_{1}}, \ldots, \sigma_{a_{n}}, \tau_{1,1}, \ldots, \tau_{1, i_{1}}, \ldots, \tau_{p, 1}, \ldots, \tau_{p, i_{p}}$ run over $G_{0}$,

be a finite subset of this type.

Let $j, 1 \leq j \leq p$. Then $\operatorname{card}\left(\Gamma_{a_{n+j}}\right)>1$. By hypothesis, $\operatorname{card}\left(\Gamma_{a_{n+j}}^{\prime}\right)>1$, so $\Gamma_{a_{n+j}}^{\prime}$ is infinite.

Hence there exists $\tau_{j}^{\prime}$ in $G^{\prime}$ such that:

$$
\neg g^{\prime}\left(a_{n+j}, \tau_{j}^{\prime}-f_{0}\left(\tau_{j, 1}\right)\right), \ldots, \neg g^{\prime}\left(a_{n+j}, \tau_{j}^{\prime}-f_{0}\left(\tau_{j, i_{j}}\right)\right) .
$$

Now, $G^{\prime}$ is rich and $\kappa^{+}$-saturated, hence there exists $\sigma^{\prime}$ in $G^{\prime}$ such that:

$$
\bigwedge_{a \in I_{\mathrm{y}}} g^{\prime}\left(a, \sigma^{\prime}-f_{0}\left(\sigma_{a}\right)\right) \text { and } \bigwedge_{a \in I_{\mathrm{n}}} \bigwedge_{\tau \in G_{0}} \neg g^{\prime}\left(a, \sigma^{\prime}-f_{0}(\tau)\right) \text {. }
$$

Since $\forall \tau^{\prime} \in G_{0}^{\prime}, \neg g\left(a_{1}, \sigma^{\prime}-\tau^{\prime}\right) \Rightarrow \forall \tau^{\prime} \in G_{0}^{\prime}, \sigma^{\prime} \neq \tau^{\prime}$, if $I_{\mathrm{n}} \neq \varnothing$, then $\sigma^{\prime} \notin G_{0}^{\prime}$. We set $f_{1}(\sigma):=\sigma^{\prime}$.

If $I_{\mathrm{n}}=\varnothing$, and $\sigma^{\prime} \in G_{0}^{\prime}$, then $\sigma-f_{0}^{-1}\left(\sigma^{\prime}\right) \in G_{\mathrm{n}}$. Hence $G_{\mathrm{n}}$ is not trivial, and it follows that $\operatorname{card}\left(G_{\mathrm{n}}^{\prime}\right)>\kappa$. We let $f_{1}(\sigma)$ be any element of $\left(\sigma^{\prime}+G_{\mathrm{n}}^{\prime}\right) \backslash G_{0}^{\prime}$.

Let $G_{1}^{\prime}$ be the divisible hull of the subgroup generated by $\sigma^{\prime}+G_{0}^{\prime}$. We have: $G_{1} \simeq \mathbb{Q} \sigma \oplus G_{0}$ and $G_{1}^{\prime} \simeq \mathbb{Q} f_{1}(\sigma) \oplus G_{0}^{\prime}$ (where $\mathbb{Q}$ is the set of all rational numbers). For every $r \in \mathbb{Q}$ and $\tau \in G_{0}$, set $f_{1}(r \sigma+\tau)=r f_{1}(\sigma)+f_{0}(\tau)$. Then $f_{1}$ is an isomorphism from the group $G_{1}$ onto the group $G_{1}^{\prime}$, that extends $f_{0}$. Let $r \in \mathbb{Q} \backslash\{0\}, \tau \in G_{0}$ and $a \in I$ such that $g(a, r \sigma+\tau)$. Since $G_{a}$ is divisible, $g\left(a, \sigma+r^{-1} \tau\right)$ holds. Hence $g^{\prime}\left(a, f_{1}(\sigma)+r^{-1} f_{0}(\tau)\right)$ holds. Therefore $g^{\prime}\left(a, r f_{1}(\sigma)+f_{0}(\tau)\right)$ holds, that is $g^{\prime}\left(a, f_{1}(r \sigma+\tau)\right)$ holds. In the same way, $g^{\prime}\left(a, f_{1}(r \sigma+\tau)\right) \Rightarrow g(a, r \sigma+\tau)$. Thus, $f_{1}$ is a $\mathcal{L} g_{I}$-isomorphism.

3.5. Existentially equivalent cyclically valued groups. Let us consider the language $\mathcal{L}_{g, C}=(+, 0, C,(\cdot, \cdot, \cdot), g(\cdot, \cdot)$ The theory of cyclically valued groups $(G,+, v)$ such that $v$ is support-definable has the following set of axioms.

$\forall a, \forall b, \forall c,(a, b, c) \Rightarrow C(a) \& C(b) \& C(c) \& a \neq b \neq c \neq a \&(b, c, a) \& \neg(a, c, b)$

$\forall a, \forall b, \forall c, C(a) \& C(b) \& C(c) \& a \neq b \neq c \neq a \& \neg(a, b, c) \Rightarrow(a, c, b)$

$\forall a, \forall b, \forall c, \forall d,(a, b, d) \&(a, d, c) \Rightarrow(a, b, c)$

$\neg C(0)$

$\forall \sigma, \forall \tau, \forall \rho, \sigma+\tau=\rho \Rightarrow \neg C(\sigma) \wedge \neg C(\tau) \wedge \neg C(\rho) \wedge \tau+\sigma=\rho$

$\forall \sigma, \neg C(\sigma) \Rightarrow \sigma+0=\sigma$

$\forall \sigma, \neg C(\sigma) \Rightarrow \exists \sigma^{\prime}, \sigma+\sigma^{\prime}=0$

$\forall \sigma, \forall \tau, \forall \rho, \neg C(\sigma), \neg C(\tau), \neg C(\rho) \Rightarrow(\sigma+\tau)+\rho=\sigma+(\tau+\rho)$

$\forall a, \forall \sigma, g(a, \sigma) \Rightarrow C(a) \& \neg C(\sigma)$

$\forall a, C(a) \Rightarrow g(a, 0)$

$\forall a, \forall \sigma, \forall \tau, g(a, \sigma) \& g(a, \tau) \Rightarrow g(a, \tau+\sigma)$ 
$\forall a, \forall \sigma, \forall \tau, g(a, \sigma) \& \sigma+\tau=0 \Rightarrow g(a, \tau)$

$\forall \sigma, \neg C(\sigma) \& \sigma \neq 0 \Rightarrow$

$[\forall a, C(a) \Rightarrow[\neg(g(a, \sigma)$ or $[\exists b, \neg g(b, \sigma) \& \forall c,(a, c, b) \Rightarrow g(c, \sigma)]]]$.

Note that as a consequence of the last axiom we have: $\forall \sigma,(\neg C(\sigma) \& \forall a, C(a) \Rightarrow g(a, \sigma)) \Leftrightarrow \sigma=0$.

A structure for the language $\mathcal{L}_{g, C}$ will be denoted by $(G,+, g, C)$, or $(G,+, g)$.

Definition 3.5.1. Let $(G,+, v)$ be a cyclically valued group. We will say that $(G,+, v)$ is $r i c h$ if $v$ is support-definable and the family $(g(a, \cdot), a \in C)$, defined in subsection 3.4 , is rich.

Remark 3.5.2. By Remark 3.4.5, every cyclically valued group admits a rich closure. This closure is defined in the same way as in Proposition 3.4.4. The rich closure can be called "canonical" because any isomorphism from a cyclically valued group onto cyclically valued group extends to the rich closures.

$(G,+, g)$ is rich if $v$ is support-definable and for every pair $(n, p)$ of positive integers and for every $p$-uple $\left(i_{1}, \ldots, i_{p}\right)$ of integers, it satisfies the following formulas:

$$
\begin{gathered}
\forall a_{1}, \ldots, \forall a_{n}, \forall a_{n+1}, \ldots, \forall a_{n+p}, \forall \sigma_{1}, \ldots, \forall \sigma_{n}, \forall \tau_{1,1}, \ldots, \forall \tau_{1, i_{1}}, \ldots, \forall \tau_{p, 1}, \ldots, \forall \tau_{p, i_{p}}, \\
C\left(a_{1}\right) \& \ldots \& C\left(a_{n}\right) \& C\left(a_{n+1}\right) \& \ldots \& C\left(a_{n+p}\right) \& \bigwedge_{i \neq j} a_{i} \neq a_{j} \\
\& \neg C\left(\sigma_{1}\right) \& \ldots \& \neg C\left(\sigma_{n}\right) \& \neg C\left(\tau_{1,1}\right) \& \ldots \& \neg C\left(\tau_{1, i_{1}}\right) \& \ldots \& \neg C\left(\tau_{p, 1}\right) \& \ldots \\
\& \neg C\left(\tau_{p, i_{p}}\right) \& \exists \tau_{1}, \ldots, \exists \tau_{p}, \neg C\left(\tau_{1}\right) \& \ldots \& \neg C\left(\tau_{p}\right) \& \\
\neg g\left(a_{n+1}, \tau_{1}-\tau_{1,1}\right), \ldots, \neg g\left(a_{n+1}, \tau_{1}-\tau_{1, i_{1}}\right), \ldots, \neg g\left(a_{n+p}, \tau_{p}-\tau_{p, 1}\right), \ldots, \neg g\left(a_{n+p}, \tau_{p}-\tau_{p, i_{p}}\right) \Rightarrow \\
\exists \sigma, g\left(a_{1}, \sigma_{1}-\sigma\right), \ldots, g\left(a_{n}, \sigma_{n}-\sigma\right), \neg g\left(a_{n+1}, \sigma-\tau_{1,1}\right), \ldots, \neg g\left(a_{n+1}, \sigma-\tau_{1, i_{1}}\right), \ldots, \\
\neg g\left(a_{n+p}, \sigma-\tau_{p, 1}\right), \ldots, \neg g\left(a_{n+p}, \sigma-\tau_{p, i_{p}}\right) .
\end{gathered}
$$

Theorem 3.5.3. Let $(G,+, g)$ and $\left(G^{\prime},+, g^{\prime}\right)$ be two rich cyclically valued groups. Assume that: both of $(G,+)$ and $\left(G^{\prime},+\right)$ are both torsion-free and divisible, for every $a \in I$, both of $\{\sigma \in G \mid g(a, \sigma)\}$ and $\left\{\sigma^{\prime} \in G^{\prime} \mid g^{\prime}\left(a, \sigma^{\prime}\right)\right\}$ are divisible, for every $a \in I, G=\{\sigma \in G \mid g(a, \sigma)\} \Leftrightarrow G^{\prime}=\left\{\sigma^{\prime} \in G^{\prime} \mid g^{\prime}\left(a, \sigma^{\prime}\right)\right\}$.

Let $n, p$ be two integers. For $1 \leq i \leq n+p$, let $Q_{i} \in\{\forall, \exists\}$, and let $\varphi\left(a_{1}, \ldots, a_{n}, \sigma_{1}, \ldots, \sigma_{p}\right)$ be a quantifier-free formula for the language $\mathcal{L}_{g, C}$. Then:

$$
\begin{gathered}
(G,+, g) \models Q_{1} a_{1}, \ldots, Q_{n} a_{n}, Q_{n+1} \sigma_{1}, \ldots, Q_{n+p} \sigma_{p}, \bigwedge_{1 \leq i \leq n} C\left(a_{i}\right) \\
\& \bigwedge_{1 \leq i \leq p} \neg C\left(\sigma_{i}\right) \& \varphi\left(a_{1}, \ldots, a_{n}, \sigma_{1}, \ldots, \sigma_{p}\right)
\end{gathered}
$$

if and only if

$$
\begin{gathered}
\left(G^{\prime},+, g^{\prime}\right) \models Q_{1} a_{1}, \ldots, Q_{n} a_{n}, Q_{n+1} \sigma_{1}, \ldots, Q_{n+p} \sigma_{p}, \bigwedge_{1 \leq i \leq n} C\left(a_{i}\right) \\
\& \bigwedge_{1 \leq i \leq p} \neg C\left(\sigma_{i}\right) \& \varphi\left(a_{1}, \ldots, a_{n}, \sigma_{1}, \ldots, \sigma_{p}\right) .
\end{gathered}
$$

In particular, $(G,+, g) \equiv_{\exists}\left(G^{\prime},+, g^{\prime}\right)$.

Proof. We can assume that $\varphi\left(a_{1}, \ldots, a_{n}, \sigma_{1}, \ldots, \sigma_{p}\right)$ is a disjunction of conjunctions of atomic formulas, say:

$$
\varphi\left(a_{1}, \ldots, a_{n}, \sigma_{1}, \ldots, \sigma_{p}\right)=\theta_{1}\left(a_{1}, \ldots, a_{n}, \sigma_{1}, \ldots, \sigma_{p}\right) \vee \cdots \vee \theta_{m}\left(a_{1}, \ldots, a_{n}, \sigma_{1}, \ldots, \sigma_{p}\right),
$$

where every $\theta_{l}$ is a conjunction:

$$
\begin{gathered}
\bigwedge_{N_{1}}\left(a_{i}, a_{j}, a_{k}\right) \& \bigwedge_{N_{2}} \neg\left(a_{i}, a_{j}, a_{k}\right) \& \bigwedge_{N_{3}} a_{i}=a_{j} \& \bigwedge_{N_{4}} a_{i} \neq a_{j} \& \bigwedge_{N_{5}} g\left(a_{i}, \sigma_{j}-\sigma_{k}\right) \\
\& \bigwedge_{N_{6}} \neg g\left(a_{i}, \sigma_{j}-\sigma_{k}\right) \& \bigwedge_{N_{7}} \sigma_{i} \neq \sigma_{j} \& \bigwedge_{N_{8}} \sigma_{i} \neq \sigma_{j} \\
\& \bigwedge_{N_{9}} P_{i}\left(\sigma_{1}, \ldots, \sigma_{p}\right)=Q_{i}\left(\sigma_{1}, \ldots, \sigma_{p}\right), \& \bigwedge_{N_{10}} P_{i}^{\prime}\left(\sigma_{1}, \ldots, \sigma_{p}\right) \neq Q_{i}^{\prime}\left(\sigma_{1}, \ldots, \sigma_{p}\right) \\
\text { where } P_{i}, Q_{i}, P_{i}^{\prime}, Q_{i}^{\prime} \text { belong to } \mathbb{Z}\left[X_{1}, \ldots, X_{p}\right] .
\end{gathered}
$$


Let $\left(c_{1}, \ldots, c_{n}\right)$ be a $n$-tuple of elements of $C$. Then either:

or:

$$
(C,(\cdot, \cdot, \cdot)) \models \bigwedge_{N_{1}}\left(c_{i}, c_{j}, c_{k}\right) \& \bigwedge_{N_{2}} \neg\left(c_{i}, c_{j}, c_{k}\right) \& \bigwedge_{N_{3}} c_{i}=c_{j} \& \bigwedge_{N_{4}} c_{i} \neq c_{j},
$$

$$
(C,(\cdot, \cdot, \cdot)) \forall \bigwedge_{N_{1}}\left(c_{i}, c_{j}, c_{k}\right) \& \bigwedge_{N_{2}} \neg\left(c_{i}, c_{j}, c_{k}\right) \& \bigwedge_{N_{3}} c_{i}=c_{j} \& \bigwedge_{N_{4}} c_{i} \neq c_{j} .
$$

In the former case, $\theta_{l}\left(c_{1}, \ldots, c_{n}, \sigma_{1}, \ldots, \sigma_{p}\right)$, is equivalent to:

$$
\begin{gathered}
\bigwedge_{N_{5}} g\left(c_{i}, \sigma_{j}-\sigma_{k}\right) \& \bigwedge_{N_{6}} \neg g\left(c_{i}, \sigma_{j}-\sigma_{k}\right) \& \bigwedge_{N_{7}} \sigma_{i} \neq \sigma_{j} \& \bigwedge_{N_{8}} \sigma_{i} \neq \sigma_{j} \\
\& \bigwedge_{N_{9}} P_{i}\left(\sigma_{1}, \ldots, \sigma_{p}\right)=Q_{i}\left(\sigma_{1}, \ldots, \sigma_{p}\right) \& \bigwedge_{N_{10}} P_{i}^{\prime}\left(\sigma_{1}, \ldots, \sigma_{p}\right) \neq Q_{i}^{\prime}\left(\sigma_{1}, \ldots, \sigma_{p}\right),
\end{gathered}
$$

and in the latter case, $\theta_{l}\left(c_{1}, \ldots, c_{n}, \sigma_{1}, \ldots, \sigma_{p}\right)$ doesn't hold for any model.

Therefore, $\varphi\left(c_{1}, \ldots, c_{n}, \sigma_{1}, \ldots, \sigma_{p}\right)$ is equivalent to a formula of the language $\left(+, 0, g\left(c_{i}, \cdot\right), 1 \leq i \leq n\right)$. Then by Theorem 3.4.7,

$$
(G,+, g) \models Q_{n+1} \sigma_{1}, \ldots, Q_{n+p} \sigma_{p}, \bigwedge_{1 \leq i \leq p} \neg C\left(\sigma_{i}\right) \& \varphi\left(c_{1}, \ldots, c_{n}, \sigma_{1}, \ldots, \sigma_{p}\right)
$$

if and only if

$$
\left(G^{\prime},+, g^{\prime}\right) \models Q_{n+1} \sigma_{1}, \ldots, Q_{n+p} \sigma_{p}, \bigwedge_{1 \leq i \leq p} \neg C\left(\sigma_{i}\right) \& \varphi\left(c_{1}, \ldots, c_{n}, \sigma_{1}, \ldots, \sigma_{p}\right) .
$$

We have proved

$$
\begin{aligned}
(G,+, g) & \models Q_{1} a_{1}, \ldots, Q_{n} a_{n}, Q_{n+1} \sigma_{1}, \ldots, Q_{n+p} \sigma_{p}, \bigwedge_{1 \leq i \leq n} C\left(a_{i}\right) \\
& \& \bigwedge_{1 \leq i \leq p} \neg C\left(\sigma_{i}\right) \& \varphi\left(a_{1}, \ldots, a_{n}, \sigma_{1}, \ldots, \sigma_{p}\right)
\end{aligned}
$$

if and only if

$$
\begin{gathered}
\left(G^{\prime},+, g^{\prime}\right) \models Q_{1} a_{1}, \ldots, Q_{n} a_{n}, Q_{n+1} \sigma_{1}, \ldots, Q_{n+p} \sigma_{p}, \bigwedge_{1 \leq i \leq n} C\left(a_{i}\right) \\
\& \bigwedge_{1 \leq i \leq p} \neg C\left(\sigma_{i}\right) \& \varphi\left(a_{1}, \ldots, a_{n}, \sigma_{1}, \ldots, \sigma_{p}\right) .
\end{gathered}
$$

Any existential formula is equivalent to a formula

$$
\exists a_{1}, \ldots, \exists a_{n}, \exists \sigma_{1}, \ldots, \exists \sigma_{p}, \varphi\left(a_{1}, \ldots, a_{n}, \sigma_{1}, \sigma_{p}\right),
$$

where $\varphi$ is a quantifier-free formula.

Now, $(G,+, g)$ is a model of this formula if and only if $\left(G^{\prime},+, g^{\prime}\right)$ is. Thus $(G,+, g) \equiv_{\exists}\left(G^{\prime},+, g^{\prime}\right)$.

Once again, this result doesn't hold if we take $\equiv$ instead of $\equiv_{\exists}$. For example, assume that $C$ is both infinite and well-ordered. For every $a \in C$, let $\Gamma_{a}$ be a non-trivial, divisible, torsion-free, abelian group. Set $G:=\sum_{a \in C} \Gamma_{a}$ and $G^{\prime}:=\prod_{a \in C} \Gamma_{a}$. Then $G^{\prime}$ enjoys $\forall \sigma, \exists \tau, \forall a, C(a) \Rightarrow \neg g(a, \sigma-\tau)$, but $G$ doesn't. Theorem 4. Let $(G,+, g, C)$ and $\left(G^{\prime},+, g^{\prime}, C^{\prime}\right)$ be two rich cyclically valued groups such that $C \equiv C^{\prime}$. Assume that:

both of $(G,+)$ and $\left(G^{\prime},+\right)$ are both torsion-free and divisible, for every $a \in I$, both of $\{\sigma \in G \mid g(a, \sigma)\}$ and $\left\{\sigma^{\prime} \in G^{\prime} \mid g^{\prime}\left(a, \sigma^{\prime}\right)\right\}$ are divisible, for every $a$ in $C$ and for every $a^{\prime}$ in $C^{\prime}, G=\left\{\sigma \in G \mid g(a, \sigma\} \Leftrightarrow G^{\prime}=\left\{\sigma^{\prime} \in G^{\prime} \mid g^{\prime}\left(a^{\prime}, \sigma\right\}\right.\right.$.

Let $n, p$ be two integers. For $1 \leq i \leq n+p$, let $Q_{i} \in\{\forall, \exists\}$, and let $\varphi\left(a_{1}, \ldots, a_{n}, \sigma_{1}, \ldots, \sigma_{p}\right)$ be $a$ quantifier-free formula for the language $(+, 0,(\cdot, \cdot, \cdot), g(a, \cdot))$. Then:

$$
\begin{gathered}
(G,+, g, C) \models Q_{1} a_{1}, \ldots, Q_{n} a_{n}, Q_{n+1} \sigma_{1}, \ldots, Q_{n+p} \sigma_{p}, \bigwedge_{1 \leq i \leq n} C\left(a_{i}\right) \\
\& \bigwedge_{1 \leq i \leq p} \neg C\left(\sigma_{i}\right) \& \varphi\left(a_{1}, \ldots, a_{n}, \sigma_{1}, \ldots, \sigma_{p}\right)
\end{gathered}
$$


if and only if

$$
\begin{gathered}
\left(G^{\prime},+, g^{\prime}, C^{\prime}\right) \models Q_{1} a_{1}, \ldots, Q_{n} a_{n}, Q_{n+1} \sigma_{1}, \ldots, Q_{n+p} \sigma_{p}, \\
\bigwedge_{1 \leq i \leq n} C\left(a_{i}\right) \& \bigwedge_{1 \leq i \leq p} \neg C\left(\sigma_{i}\right) \& \varphi\left(a_{1}, \ldots, a_{n}, \sigma_{1}, \ldots, \sigma_{p}\right) .
\end{gathered}
$$

In particular, $(G,+, g, C) \equiv_{\exists}\left(G^{\prime},+, g^{\prime}, C^{\prime}\right)$.

Proof. It is similar to the proof of Theorem 3.

\section{From Ultrametric Distances to CyCLIC ONES.}

4.1. Dedekind cuts of cyclically ordered sets. We will say that $\varpi=(C,<)$ is a Dedekind cut of $(C,(\cdot, \cdot, \cdot))$ if $(\cdot, \cdot, \cdot)$ is the cyclic order defined by $<$ (see [No 84] and [Nos 87]). The total order corresponding to the Dedekind cut $\varpi$ will be denoted by $<_{\varpi}$. We will say that $\varpi$ is a principal Dedekind cut if the order $<_{\varpi}$ is equal to an order $<_{c}$, for some $c \in C$, .

Let $\varpi$ be a Dedekind cut of $(C,(\cdot, \cdot, \cdot))$. We can extend the total order $<_{\varpi}$ to a total order $<^{\prime}$ over $C \cup\{\varpi\}$ by setting: $\forall c \in C, \varpi<^{\prime} c$. Let $(\cdot, \cdot, \cdot)^{\prime}$ be the cyclic order over $C \cup\{\varpi\}$ defined by $<^{\prime}$. Then $(\cdot, \cdot, \cdot)^{\prime}$ extends $(\cdot, \cdot, \cdot)$ to $C \cup\{\varpi\}$.

Proposition 4.1. Let $(E, O, u)$ be a cyclically ultrametric space such that $u$ is support-definable, and let $\varpi$ be a non-principal Dedekind cut of $C$.

If $\min _{\varpi}\{u(a, \sigma, \tau) \mid a \in C\}$ exists, then set $u(\varpi, \sigma, \tau):=\min _{\varpi}\{u(a, \sigma, \tau) \mid a \in C\}$;

otherwise, set $u(\varpi, \sigma, \tau):=\varpi$.

Extend the cyclic order $(\cdot, \cdot, \cdot)$ to $C \cup\{\varpi\}$. Let $\sigma, \tau$ in $E$. Set $u^{\prime}(\varpi, \sigma, \tau):=u(\varpi, \sigma, \tau)$, and for $a \in C$, set $u^{\prime}(a, \sigma, \tau):=u(a, \sigma, \tau)$.

Then $u^{\prime}: C \cup\{\varpi\} \times E \times E \rightarrow C \cup\{\varpi\}$ is a cyclic ultrametric distance which is defined by the supports.

Proof.

We have: $u^{\prime}(\varpi, \sigma, \tau)=\infty \Leftrightarrow \min _{a}\{u(a, \sigma, \tau) \mid a \in C\}=\infty \Leftrightarrow \forall a \in C, u(a, \sigma, \tau)=\infty \Leftrightarrow \sigma=\tau$.

Let $\sigma, \tau, \rho$ in $E$. Set $a_{0}=u^{\prime}(\varpi, \sigma, \tau), a_{1}=u^{\prime}(\varpi, \sigma, \rho), a_{2}=u^{\prime}(\varpi, \rho, \tau)$. If $\varpi=\min _{\varpi}\left(a_{1}, a_{2}\right)$, then trivially $a_{0} \geq_{\varpi} \min _{\varpi}\left(a_{1}, a_{2}\right)$. Assume that $\varpi<_{\varpi} \min _{\varpi}\left(a_{1}, a_{2}\right)$, and let $a \in C, a \leq_{\varpi} \min _{\varpi}\left(a_{1}, a_{2}\right)$. Then $u(a, \sigma, \tau) \geq_{a} \min _{a}(u(a, \sigma, \rho), u(a, \rho, \tau))=\min _{a}\left(a_{1}, a_{2}\right)$. By the definition of $u^{\prime}(\varpi, \cdot, \cdot)$, we have $a_{0} \geq_{\varpi} \min _{\varpi}\left(a_{1}, a_{2}\right)$. Hence $u^{\prime}(\varpi, \cdot, \cdot)$ is an ultrametric distance.

Let $\sigma, \tau$ in $E$. Set $\operatorname{Supp}(\sigma, \tau):=\{u(a, \sigma, \tau) \mid a \in C\}$ and $\operatorname{Supp}^{\prime}(\sigma, \tau):=\left\{u^{\prime}(a, \sigma, \tau) \mid a \in C \cup\{\varpi\}\right\}$.

If $\varpi \notin \operatorname{Supp}^{\prime}(\sigma, \tau)$, then $\operatorname{Supp}(\sigma, \tau)=\operatorname{Supp}^{\prime}(\sigma, \tau)$. So, for any $a \in C, \min _{a} \operatorname{Supp}^{\prime}(\sigma, \tau)=u^{\prime}(a, \sigma, \tau)$. Furthermore, $u^{\prime}(\varpi, \sigma, \tau)=\min _{\varpi} \operatorname{Supp}(\sigma, \tau)=\min _{\varpi} \operatorname{Supp}^{\prime}(\sigma, \tau)$.

Assume that $\varpi \in \operatorname{Supp}^{\prime}(\sigma, \tau)$. Then $\operatorname{Supp}(\sigma, \tau)$ is coinitial in $\left(C, \leq_{\varpi}\right)$. Now, for every $a \in C$, $\min _{a} \operatorname{Supp}(\sigma, \tau)=u(a, \sigma, \tau)$ exists. Hence $u(a, \sigma, \tau)<_{a} \varpi$, and $u^{\prime}(a, \sigma, \tau)=u(a, \sigma, \tau)=\min _{a} \operatorname{Supp}(\sigma, \tau)=$ $\min _{a} \operatorname{Supp}^{\prime}(\sigma, \tau)$.

4.2. Ultrametric distances. In [P-R 96] the authors proved that every generalized ultrametric space can be embedded into a Hahn product. When applied to "classical" ultrametric spaces, this embedding gives rise to a cyclic ultrametric distance over $E$. But in general, this Hahn product needs not be an immediate extension of $E$ (see Definition 4.2).

Let $(E, \nu)$ be an ultrametric space, and $T:=\nu(E \times E)$. For every $\sigma \in E$ and $t \in T$, the set $\left\{\sigma^{\prime} \in E \mid \nu\left(\sigma, \sigma^{\prime}\right)>t\right\}$ will be called the open ball of center $\sigma$ and radius $t$, and it will be denoted by $B_{o}(\sigma, t)$. The set $\left\{\sigma^{\prime} \in E \mid \nu\left(\sigma, \sigma^{\prime}\right) \geq t\right\}$ will be called the closed ball of center $\sigma$ and radius $t$, and it will be denoted by $B_{c}(\sigma, t)$.

Let $\left(\Gamma_{a}\right)_{a \in C}$ be a family of non-empty sets. For every $\sigma=\left(\sigma_{a}\right)_{a \in C}, \tau=\left(\tau_{a}\right)_{a \in C}$ in $\prod_{a \in C} \Gamma_{a}$, denote by $\operatorname{Supp}(\sigma, \tau)$ the set $\left\{a \in C \mid \sigma_{a} \neq \tau_{a}\right\}$. For every $a \in C$, we let $O_{a}$ be a fixed element of $\Gamma_{a}$, and we set $O:=\left(O_{a}\right)_{a \in C} \in \prod_{a \in C} \Gamma_{a}$. The subset $\Pi_{a \in C} \Gamma_{a}$, which consists of all elements $\sigma$ such that $\operatorname{Supp}(\sigma, O)$ is well-ordered, will be called the Hahn product of the $\Gamma_{a}$ 's. The Hahn products satisfy condition $(*)$ of Proposition 2.3.

Theorem 5. Let $(E, \nu)$ be an ultrametric space, $T:=\nu(E \times E),(\cdot, \cdot, \cdot)$ be the cyclic order defined by $<$. Then there exists a cyclic ultrametric distance $u$ on $E$, such that $u$ is defined by the supports and for every $\sigma$ and $\tau$ in E, Supp $(\sigma, \tau)$ is well-ordered. Furthermore, if $\varpi$ is the Dedekind cut $(T,<)$ of $(T,(\cdot, \cdot, \cdot))$, then $u(\varpi, \cdot, \cdot)$ is equal to $\nu$. 
Proof. In order to prove this theorem, we will show that the ultrametric space $(E, \nu)$ embeds into a Hahn product $\Pi_{t \in T}\left(\{O\} \cup M_{t}\right)$, where $O$ is a fixed element of $E$, such that the image of $O$ is $(O)_{t \in T}$. If $(E, \nu)$ is a valued field, every $\{O\} \cup M_{t}$ can be taken isomorphic to the residue field (see [Ri 64], proof of a lemma of Krull, pp. 80-87). Now, in the general case, we have to define the sets $M_{t}$.

For every $\sigma \in E$ and $t \in T$, we will denote by $\mathcal{B}(\sigma, t)$ the set of all open balls of radius $t$ contained in the closed ball $B_{c}(\sigma, t)$. $(E, \nu)$ being an ultrametric space, we know that for every $\sigma_{1}, \sigma_{2}$ in $E$ and $t_{1} \leq t_{2}$ in $T$, either $B_{o}\left(\sigma_{2}, t_{2}\right) \subset B_{o}\left(\sigma_{1}, t_{1}\right)$, or $B_{o}\left(\sigma_{1}, t_{1}\right) \cap B_{o}\left(\sigma_{2}, t_{2}\right)=\varnothing$ (the same holds by taking closed balls instead of open balls). It follows that for every $\sigma \neq O$, there is a unique ball $B$ in $\mathcal{B}(O, \nu(O, \sigma)) \backslash\left\{B_{o}(O, \nu(O, \sigma))\right\}$ such that $\sigma \in B$. If $t<\nu(O, \sigma)$, then $\sigma \in B_{o}(O, t)$. If $t>\nu(O, \sigma)$, then $\forall B \in \mathcal{B}(O, t), \sigma \notin B$. For every $t$ in $T$, and $B$ in $\mathcal{B}(O, t) \backslash\left\{B_{o}(O, t)\right\}$, we take an element $\rho_{B}$ of $B$. If for all $\sigma \in E, \operatorname{card}(\mathcal{B}(\sigma, t)) \leq \operatorname{card}(\mathcal{B}(O, t))$, then we set $M_{t}:=\left\{\rho_{B} \mid B \in \mathcal{B}(O, t) \backslash\left\{B_{o}(O, t)\right\}\right\}$. Otherwise, $M_{t}$ is an arbitrary set satisfying:

$M_{t} \cap E=\left\{\rho_{B} \mid B \in \mathcal{B}(O, t) \backslash\left\{B_{o}(O, t)\right\}\right\}$

$\rho \in M_{t} \backslash E \Rightarrow \exists \sigma \in E, \operatorname{card}(\mathcal{B}(\sigma, t))>\operatorname{card}(\mathcal{B}(O, t))$, and $\rho \in \mathcal{B}(\sigma, t) \times\{t\}$

$1+\operatorname{card}\left(M_{t}\right)$ is the union of the cardinals of all the $\mathcal{B}(\sigma, t), \sigma \in E$.

Observe that for $B_{1} \neq B_{2}$ in $\mathcal{B}(O, t) \backslash\left\{B_{o}(O, t)\right\}, \rho_{B_{1}} \neq \rho_{B_{2}}$, and that $M_{t_{1}} \cap M_{t_{2}}=\varnothing$ whenever $t_{1} \neq t_{2}$.

We set $f_{0}(O):=(O)_{t \in T}$, and for $t \in T, \rho \in M_{t} \cap E, f_{0}(\rho):=\left(f_{0}(\rho)_{t^{\prime}}\right)_{t^{\prime} \in T}$ where $f_{0}(\rho)_{t}=\rho$ and $f_{0}(\rho)_{t^{\prime}}=O$ if $t^{\prime} \neq t$. Then straightforward checking shows that $f_{0}$ is an isomorphism from the ultrametric space $\{O\} \cup \bigcup_{t \in T}\left(M_{t} \cap E\right)$ into a subspace of $\sqcap_{t \in T}\left(\{O\} \cup M_{t}\right)$.

Let $\mathcal{E}$ be the set of all couples $(F, f)$ such that:

(a) $\{O\} \cup \bigcup_{t \in T}\left(M_{t} \cap E\right) \subset F \subset E$,

(b) $f$ is an isomorphism from the ultrametric space $F$ into $\sqcap_{t \in T}\left(\{O\} \cup M_{t}\right)$ which extends $f_{0}$,

(c) for every $\sigma_{0} \in F, t \in T$ there exists a one-to-one mapping $\varphi_{F, f, B_{o}\left(\sigma_{0}, t\right)}$ from $\mathcal{B}\left(\sigma_{0}, t\right)$ into $\{O\} \cup M_{t}$, which extends the mapping:

$$
\left\{\begin{array}{cl}
\left\{B_{o}(\sigma, t) \mid \sigma \in F \text { and } \nu\left(\sigma, \sigma_{0}\right)=t\right\} & \rightarrow\{O\} \cup M_{t} \\
B_{o}(\sigma, t) & \mapsto f(\sigma)_{t}
\end{array}\right.
$$

Note that for $\sigma_{1}, \sigma_{2}$ in $F$ with $\nu\left(\sigma_{1}, \sigma_{2}\right)=t_{0}$, then $B_{o}\left(\sigma_{1}, t_{0}\right) \neq B_{o}\left(\sigma_{2}, t_{0}\right)$. Thus $f\left(\sigma_{1}\right)_{t_{0}} \neq f\left(\sigma_{2}\right)_{t_{0}}$. Now, for all $t<t_{0}, B_{o}\left(\sigma_{1}, t\right)=B_{o}\left(\sigma_{2}, t\right)$. Hence $f\left(\sigma_{1}\right)_{t}=f\left(\sigma_{2}\right)_{t}$.

Trivially, $\left(\{O\} \cup \bigcup_{t \in T}\left(M_{t} \cap E\right), f_{0}\right)$ belongs to $\mathcal{E}$. So $\mathcal{E}$ is nonempty.

Let $(F, f)$ and $\left(F^{\prime}, f^{\prime}\right)$ be elements of $\mathcal{E}$. Set $(F, f) \leq\left(F^{\prime}, f^{\prime}\right)$ if and only if $F \subset F^{\prime}, f$ is the restriction of $f^{\prime}$ to $F$ and $\forall \sigma_{0} \in F, \forall t \in T, \varphi_{F^{\prime}, f^{\prime}, B_{o}\left(\sigma_{0}, t\right)}=\varphi_{F, f, B_{o}\left(\sigma_{0}, t\right)}$.

One checks easily that $(\mathcal{E}, \leq)$ is inductively ordered. By Zorn's lemma, it contains maximal elements.

Let $(F, f) \in \mathcal{E}$, assume that there exists $\sigma \in E$ such that $\sigma \notin F$.

Let $t \in T$.

If there exists $\sigma^{\prime} \in F$ such that $\nu\left(\sigma, \sigma^{\prime}\right)>t$, then we set $f(\sigma)_{t}:=f\left(\sigma^{\prime}\right)_{t}$.

If for all $\sigma^{\prime} \in F, \nu\left(\sigma, \sigma^{\prime}\right)<t$, then we set $f(\sigma)_{t}:=O$.

Assume that, for all $\sigma^{\prime}$ in $F, \nu\left(\sigma^{\prime}, \sigma\right) \leq t$, and that there exists $\sigma_{0}$ in $F$ such that $\nu\left(\sigma, \sigma_{0}\right)=t$. Then $B_{o}(\sigma, t) \cap F=\varnothing$. We set $f(\sigma)_{t}:=\varphi_{F, f, B_{o}(\sigma, t)}$.

Clearly, $\left(f(\sigma)_{t}\right)_{t \in T} \in \prod_{t \in T}\{O\} \cup M_{t}$. In order to prove that $\left(f(\sigma)_{t}\right)_{t \in T} \in \sqcap_{t \in T}\{O\} \cup M_{t}$, let $S \subset \operatorname{Supp}(\sigma, O)$. If $S$ is finite, then it contains a lowest element. Assume that $S$ is infinite. Take $t_{0} \in S$ such that $t_{0}$ is not the greatest element of $S$. Then, by the definition of $f(\sigma)$, there exists $\sigma^{\prime} \in F$ such that $\nu\left(\sigma, \sigma^{\prime}\right)>t_{0}$. Hence $\left\{t \in \operatorname{Supp}(\sigma) \mid t \leq t_{0}\right\}=\left\{t \in \operatorname{Supp}\left(\sigma^{\prime}\right) \mid t \leq t_{0}\right\}$. Therefore $\left\{t \in S \mid t \leq t_{0}\right\} \subset \operatorname{Supp}\left(\sigma^{\prime}\right)$, so it is well-ordered. It follows that $\left(f(\sigma)_{t}\right)_{t \in T} \in \sqcap_{t \in T}\{O\} \cup M_{t}$.

We set $F^{\prime}:=F \cup\{\sigma\}$. Trivially, $F^{\prime}$ satisfies condition (a). Let $\sigma^{\prime} \in F$, and $t_{0}=\nu\left(\sigma, \sigma^{\prime}\right)$. It $t<t_{0}$, then $f(\sigma)_{t}=f\left(\sigma^{\prime}\right)_{t}$. If there exists $\sigma^{\prime \prime} \in F$ such that $\nu\left(\sigma, \sigma^{\prime \prime}\right)>t_{0}$, then $f(\sigma)_{t_{0}}=f\left(\sigma^{\prime \prime}\right)_{t_{0}}$. Now, $\nu\left(\sigma^{\prime}, \sigma^{\prime \prime}\right)=t_{0}$, so $f\left(\sigma^{\prime}\right)_{t_{0}} \neq f\left(\sigma^{\prime \prime}\right)_{t_{0}}$. Hence: $\nu\left(f(\sigma), f\left(\sigma^{\prime}\right)\right)=t_{0}$. If for all $\sigma^{\prime \prime} \in F, \nu\left(\sigma, \sigma^{\prime \prime}\right) \leq t_{0}$, then, by the definition of $f(\sigma)_{t_{0}}$, we have: $f(\sigma)_{t_{0}} \neq f\left(\sigma^{\prime}\right)_{t_{0}}$. It follows that $\nu\left(f(\sigma), f\left(\sigma^{\prime}\right)\right)=t_{0}=\nu\left(\sigma, \sigma^{\prime}\right)$. Consequently, for every $\sigma^{\prime} \in F, f\left(\sigma^{\prime}\right) \neq f(\sigma)$. Hence $\left(F^{\prime}, f\right)$ satisfies (b).

In order to prove (c), let $t \in T$.

If there exists $\sigma_{0} \in F$ such that $t<\nu\left(\sigma, \sigma_{0}\right)$, then $\mathcal{B}(\sigma, t)=\mathcal{B}\left(\sigma_{0}, t\right)$. We set $\varphi_{F^{\prime}, f^{\prime}, B_{o}(\sigma, t)}=$ $\varphi_{F, f, B_{o}\left(\sigma_{0}, t\right)}$, and (c) holds.

If for every $\sigma_{0} \in F, t>\nu\left(\sigma_{0}, \sigma\right)$, then $\operatorname{card}\left(\left\{B_{o}\left(\sigma^{\prime}, t\right) \mid \sigma^{\prime} \in F^{\prime}\right.\right.$ and $\left.\left.\nu\left(\sigma, \sigma^{\prime}\right)=t\right\}\right)=1$. Hence (c) 
holds.

If, for every $\sigma_{0} \in F, t \geq \nu\left(\sigma, \sigma_{0}\right)$ and there exists $\sigma_{1} \in F$ such that $\nu\left(\sigma, \sigma_{1}\right)=t$, then we set $\varphi_{F^{\prime}, f^{\prime}, B_{o}(\sigma, t)}=\varphi_{F, f, B_{o}\left(\sigma_{1}, t\right)}$. Therefore, (c) holds.

Now, if $(F, f)$ is a maximal element of $(\mathcal{E}, \leq)$, then $F=E$.

Definition 4.2. Let $(F, \nu)$ be an ultrametric space, and $E$ be a subspace of $F$. We say that the extension of ultrametric spaces $(E \subset F, \nu)$ is immediate if $\nu(E \times E)=\nu(F \times F)$ and for every $\sigma \in E$ and $\tau \in F$, with $\sigma \neq \tau$, there exists $\sigma^{\prime}$ in $E$ such that $\nu\left(\sigma^{\prime}, \tau\right)>\nu(\sigma, \tau)$.

Corollary 4.3. Hypothesis being the same as in Theorem 5, there exists an embedding $f$ from $E$ into the Hahn product $\Pi_{t \in T}\left(\{O\} \cup M_{t}\right)$ such that $\Pi_{t \in T}\left(\{O\} \cup M_{t}\right)$ is an immediate extension of $f(E)$ if and only if for every $\sigma \in E$ and $t \in T, \operatorname{card}(\mathcal{B}(\sigma, t))=\operatorname{card}(\mathcal{B}(O, t))$.

Proof. Assume that for every $\sigma \in E$ and $t \in T, \operatorname{card}(\mathcal{B}(\sigma, t))=\operatorname{card}(\mathcal{B}(O, t))$. The mappings $\varphi_{F, f, B_{o}\left(\sigma, t_{0}\right)}$ defined in the proof of Theorem 5, can be taken onto. Thus: $\forall t_{0} \in T, \forall \tau_{t_{0}} \in\{O\} \cup M_{t_{0}}, \exists \sigma, f(\sigma)_{t_{0}}=\tau_{t_{0}}$. Let $\sigma \in E, \tau \in \sqcap_{t \in T}\left(\{O\} \cup M_{t}\right)$ and set $t_{0}:=\min \operatorname{Supp}(f(\sigma), \tau)$. Then for $t<t_{0}, \tau_{t}=f(\sigma)_{t}$, and $\tau_{t_{0}} \neq f(\sigma)_{t_{0}}$. By hypothesis, there exists $B \in \mathcal{B}\left(\sigma, t_{0}\right)$ such that for every $\sigma^{\prime} \in B, f\left(\sigma^{\prime}\right)_{t_{0}}=\tau_{t_{0}}$. Now, $\forall t<t_{0}, B_{o}\left(\sigma^{\prime}, t\right)=B_{o}(\sigma, t)$, hence $f\left(\sigma^{\prime}\right)_{t}=f(\sigma)_{t}$. It follows: $\min \operatorname{Supp}\left(f\left(\sigma^{\prime}\right), \tau\right)>t_{0}$.

Now, if $\operatorname{card}\left(\mathcal{B}\left(\sigma, t_{0}\right)\right)>\operatorname{card}\left(\mathcal{B}\left(O, t_{0}\right)\right)$, take $\rho_{t_{0}}$ in $M_{t_{0}}$ such that, for all $\sigma^{\prime} \in B_{o}\left(\sigma, t_{0}\right), f\left(\sigma^{\prime}\right)_{t_{0}} \neq \rho_{t_{0}}$. For every $t<t_{0}$, set $\rho_{t}:=f(\sigma)_{t}$, and for every $t>t_{0}$, set $\rho_{t}:=O$, and let $\tau:=\left(\rho_{t}\right)_{t \in T}$. Then $\min \operatorname{Supp}(f(\sigma), \tau)=t_{0}$. Now, for every $\sigma^{\prime}$ in $E, f\left(\sigma^{\prime}\right)_{t_{0}} \neq \rho_{t_{0}}$. Hence $\min \operatorname{Supp}\left(f\left(\sigma^{\prime}\right), \tau\right) \ngtr t_{0}$.

\subsection{Valuations on groups.}

Lemma 4.4. Let $(G,+, v)$ be a cyclically valued group such that $v$ is support-definable, and let $\varpi$ be a Dedekind cut of $C$. For every $\sigma \in G \backslash\{0\}$, we set $v(\varpi, \sigma):=\min _{\varpi}(\operatorname{Supp}(\sigma))$ if this minimum exists, $v(\varpi, \sigma):=\varpi$ otherwise. Then the extension of $v$ is a cyclic valuation on $G$.

Proof. The proof is similar to the proof of Lemma 4.1.

Recall that every abelian group can be embedded in a divisible hull (see [Ku 45] or [Fu 63]). Furthermore, if $(G, \nu)$ is a (classical) valued group, then the components of $(G, \nu)$ are the quotient subgroups $\{\sigma \in G \mid \nu(\sigma) \geq t\} /\{\sigma \in G \mid \nu(\sigma)>t\}, t \in \nu(G)$.

Theorem 4.5. Let $(G, \nu)$ be an abelian valued group. Then there exists an isomorphism of valued groups from $G$ into the Hahn product of the divisible hulls of its components.

Proof. In [Co 53], Conrad deduced similar theorem in the case of torsion-free groups. Here we give a proof that includes all cases.

Let $(G, \nu)$ be a valued group. Set $T:=\nu(G)$, and for every $t \in T$, set $G_{>t}:=\{\sigma \in G \mid \nu(G)>t\}$, $G_{>t}:=\{\sigma \in G \mid \nu(G) \geq t\}$; let $\Gamma_{t}$ be the quotient group $G_{>t} / G_{>t}$, and $\tilde{\Gamma}_{t}$ be the divisible hull of $\Gamma_{t}$. We will also denote by $\nu$ the natural valuation on the Hahn product $\sqcap_{t \in T} \tilde{\Gamma}_{t}$.

We set $\mathcal{G}:=\{(H, \varphi) \mid H$ subgroup of $G, \varphi$ isomorphism of valued groups from $H$ into $\sqcap_{t \in T} \tilde{\Gamma}_{t}$, and $\left.t \in T \backslash \nu(H) \Rightarrow \forall \sigma \in H, \varphi(\sigma)_{t}=0\right\}$. $\mathcal{G}$ is nonempty, because we can take $H=\{0\}$, and $\varphi$ defined in a natural way. $\mathcal{G}$ is inductively ordered by $(H, \varphi) \leq\left(H^{\prime}, \varphi^{\prime}\right) \Leftrightarrow H \subset H^{\prime}$ and $\varphi=\varphi^{\prime}\lceil H$. According to Zorn's lemma, $\mathcal{G}$ contains maximal elements. The proof will be concluded by showing that if $(H, \varphi)$ is a maximal element of $\mathcal{G}$, then $H=G$.

Let $(H, \varphi) \in \mathcal{G}$, and assume that there exists $\sigma \in G \backslash H$. The monoid of all non-negative integers will be denoted by $\mathbb{N}$.

1) For every $n \in \mathbb{N} \backslash\{0\}$, we set $S_{n}:=\{\nu(n \sigma-\rho) \mid \rho \in H\}$. First we prove a few basic facts about the sets $S_{n}$.

a) If $t \in S_{n}$ and $t>t^{\prime} \in \nu(H)$, then $t^{\prime} \in S_{n}$. It follows that the set $\left\{S_{n} \mid n \in \mathbb{N} \backslash\{0\}\right\}$ is totally ordered by inclusion, and that if $S_{n_{1}} \subset S_{n_{2}}$, then $S_{n_{1}}$ is an initial segment of $S_{n_{2}}$.

Indeed, we have $t=\nu(n \sigma-\rho)$, for some $\rho \in H$, and $H$ contains some $\tau$ such that $\nu(\tau)=t^{\prime}$. Hence $t^{\prime}=\nu(n \sigma-(\rho+\tau)) \in S_{n}$.

b) For every $n_{1}$ and every $n_{2}$ in $\mathbb{N} \backslash\{0\}, S_{n_{1}} \cap S_{n_{2}}=S_{n_{1} \wedge n_{2}}$, where $n_{1} \wedge n_{2}$ is the gcd of $n_{1}$ and $n_{2}$. If $\nu\left(n_{1} \sigma-\rho_{1}\right) \geq t, \nu\left(n_{2} \sigma-\rho_{2}\right) \geq t$ and $u_{1} n_{1}+u_{2} n_{2}=n_{1} \wedge n_{2}$, then:

$$
\nu\left(\left(n_{1} \wedge n_{2}\right) \sigma-\left(u_{1} \rho_{1}+u_{2} \rho_{2}\right)\right)=\nu\left(u_{1} n_{1} \sigma-u_{1} \rho_{1}+u_{2} n_{2} \sigma-u_{2} \rho_{2}\right) \geq t .
$$


It follows from a) that $S_{n_{1}} \cap S_{n_{2}}$ is a subset of $S_{n_{1} \wedge n_{2}}$. If $\nu\left(\left(n_{1} \wedge n_{2}\right)-\rho\right) \geq t$, then, for $i \in\{1,2\}$, $\nu\left(n_{i}^{\prime}\left(n_{1} \wedge n_{2}\right) \sigma-n_{i}^{\prime} \rho\right) \geq t$, with $n_{i}=n_{i}^{\prime}\left(n_{1} \wedge n_{2}\right)$. Hence $S_{n_{1}} \cap S_{n_{2}} \supset S_{n_{1} \wedge n_{2}}$.

2) Now, we define $\varphi(\sigma)_{t}$, for each $t$ in $T$.

a) If $t \notin \bigcup_{n \in \mathbb{N} \backslash\{0\}} S_{n}$, we set $\varphi(\sigma)_{t}:=0$.

b) If $t \in \bigcup_{n \in \mathbb{N} \backslash\{0\}} S_{n}$, we denote by $n$ the gcd of all the integers $n$ such that $t \in S_{n}$.

i) If there exists $\rho \in H$ such that $\nu(n \sigma-\rho)>t$, we set $\varphi(\sigma)_{t}:=\frac{1}{n} \varphi(\rho)_{t}$. Assume that $\nu(n \sigma-\tau)>t$, for some $\tau \in H$. Then $\nu(\varphi(\tau)-\varphi(\rho))=\nu(\tau-\rho)>t$. So $\varphi(\tau)_{t}=\varphi(\rho)_{t}$. Therefore, $\varphi(\sigma)_{t}$ is well-defined since it is independent of the choice of $\rho$.

ii) If $t=\max S_{n}$, we take some $\rho \in H$ such that $t=\nu(n \sigma-\rho)$. Then, the class $\overline{n \sigma-\rho}$ of $n \sigma-\rho$ modulo $G_{>t}$ is not in $H_{\geq t} /\left(G_{>t} \cap H_{\geq t}\right)$ (otherwise, $t$ is not a maximal element of $\left.S_{n}\right)$. Set $\varphi(\sigma)_{t}:=$ $\frac{1}{n}\left(\overline{n \sigma-\rho}+\varphi(\rho)_{t}\right)$. Let $\tau \in H$ such that $\nu(n \sigma-\tau)=t$. Then

$$
\begin{gathered}
\overline{n \sigma-\tau}=\overline{n \sigma-\rho+\rho-\tau}=\overline{n \sigma-\rho}+\overline{\rho-\tau} . \text { Therefore, } \\
\overline{n \sigma-\tau}+\varphi(\tau)_{t}=\overline{n \sigma-\rho}+\overline{\rho-\tau}+\varphi(\tau)_{t}=\overline{n \sigma-\rho}+\varphi(\rho)_{t} .
\end{gathered}
$$

Once again, $\varphi(\sigma)_{t}$ is well-defined since it is independent of the choice of $\rho$.

3) Assume that there exists $m \in \mathbb{N} \backslash\{0\}$ and $\tau \in H$ such that $\nu(m \sigma-\tau)>t$. We prove that $\varphi(\tau)_{t}=m \varphi(\sigma)_{t}$.

Since $t \in S_{m}$, according to 1) b), there exist a lowest $n$ such that $t \in S_{n}$, and $n$ divides $m$, say $m=p n$.

a) If there exists $\rho \in H$ such that $\nu(n \sigma-\rho)>t$, then, by construction, $\varphi(\sigma)_{t}=\frac{1}{n} \varphi(\rho)_{t}$. Now, $\nu(m \sigma-\tau)>t$ and $\nu(m \sigma-p \rho)>t$. Hence $\nu(\varphi(\tau)-p \varphi(\rho))=\nu(\tau-p \rho)>t$. Therefore $\varphi(\tau)_{t}=p \varphi(\rho)_{t}=$ $\operatorname{pn\varphi }(\sigma)_{t}=m \varphi(\sigma)_{t}$.

b) If $t=\max S_{n}$, then, by 2) b), for any $\rho \in H$ such that $\nu(n \sigma-\rho)=t$, we have $\varphi(\sigma)_{t}=\frac{1}{n}(\overline{n \sigma-\rho}+$ $\left.\varphi(\rho)_{t}\right)$. Now, $\nu(\tau-p \rho)=\nu(\tau-m \sigma+p(n \sigma-\rho)) \geq t$. So $\overline{\tau-p \rho}$ is well-defined. Since $t<\nu(m \sigma-\tau)=$ $\nu(p(n \sigma-\rho)+p \rho-\tau), \overline{p(n \sigma-\rho)}=\overline{\tau-p \rho}=\varphi(\tau)_{t}-p \varphi(\rho)_{t}$. Consequently, $\varphi(\tau)_{t}=p(\overline{n \sigma-\rho})+p \varphi(\rho)_{t}=$ $m \varphi(\sigma)_{t}$.

4) Proof of: $\varphi(\sigma) \in \sqcap_{t \in T} \tilde{\Gamma}_{t}$.

a) First, we show that for every $n \in \mathbb{N} \backslash\{0\}$, $\operatorname{Supp}(\sigma) \cap S_{n}$ is well-ordered.

Let $\left(t_{i}\right)_{i<\kappa}$ be a strictly decreasing sequence of $\operatorname{Supp}(\sigma) \cap S_{n}$, and let $n_{0}$ be the lowest integer such that $\left\{t_{i} \mid i<\kappa\right\} \cap S_{n_{0}} \neq \varnothing$. By 1) a), $S_{n_{0}}$ is an initial segment of $S_{n}$. Hence there exists $\kappa^{\prime}$ such that $\left\{t_{i} \mid i<\kappa\right\} \cap S_{n_{0}}=\left\{t_{i} \mid \kappa^{\prime} \leq i<\kappa\right\}$. Let $\rho \in H$ such that $\nu\left(n_{0} \sigma-\rho\right)>t_{\kappa^{\prime}+1}$. Then the sequence $\left(t_{i}\right)_{\kappa^{\prime}+1<i<\kappa}$ is also a sequence of $\operatorname{Supp}(\rho)$. Now, $\operatorname{Supp}(\rho)$ is well-ordered, hence $\left(t_{i}\right)_{\kappa^{\prime} \leq i<\kappa}$ is finite. In the same way, one can prove, by means of a finite induction, that $\left(t_{i}\right)_{i<\kappa^{\prime}}$ is finite. Hence, the sequence $\left(t_{i}\right)_{i<\kappa}$ is finite.

b) Let $\left(t_{i}\right)_{i>\kappa}$ be a strictly decreasing sequence of $\operatorname{Supp}(\sigma)$. Then, there exists $n \in \mathbb{N} \backslash\{0\}$ such that $t_{1} \in S_{n}$. By 1) a), the sequence $\left(t_{i}\right)_{i>\kappa}$ is a sequence of $S_{n}$. Now, by a), this sequence is finite.

5) For every integer $n$, for every $\rho$ in $H$ and every $t$ in $T$, we set $\varphi(n \sigma+\rho)_{t}:=n \varphi(\sigma)_{t}+\varphi(\rho)_{t}$. Hence, $\varphi$ is a homomorphism of groups.

6) Assume that there exist $t \in T, m \in \mathbb{N} \backslash\{0\}$ and $\tau \in H$ such that $\varphi(m \sigma-\tau)_{t} \neq 0$. If $t \notin \nu(H)$, then there exist $n \in \mathbb{N} \backslash\{0\}$ and $\rho \in H$ such that $t=\nu(n \sigma-\rho)$. Hence $t \in \nu(\mathbb{Z} \sigma-\rho)$ (where $\mathbb{Z} \sigma$ is the subgroup generated by $\sigma)$. Thus: $t \in T \backslash \nu(\mathbb{Z} \sigma+H) \Rightarrow \forall m \in \mathbb{Z}, \forall \tau \in H, \varphi(m \sigma-\tau)_{t}=0$.

7) $\varphi$ is a morphism of valued groups.

Let $m \in \mathbb{N} \backslash\{0\}, \tau \in H$, and let prove that $\nu(\varphi(m \sigma-\tau))=\nu(m \sigma-\tau)$.

a) Let $t<\nu(m \sigma-\tau)$. Then, by 3), $\varphi(\tau)_{t}=m \varphi(\sigma)_{t}$. Hence $\varphi(m \sigma-\tau)_{t}=0$. It follows: $\nu(\varphi(m \sigma-\tau)) \geq$ $\nu(m \sigma-\tau)$.

b) Let $t=\nu(m \sigma-\tau)$.

i) If there exists $\rho \in H$ such that $\nu(m \sigma-\rho)>t$, then by 3), $\varphi(\rho)_{t}=m \varphi(\sigma)_{t}$. Now, $\nu(\rho-\tau)=$ $\min (\nu(\rho-m \sigma), \nu(m \sigma-\tau))=t$. Hence $\varphi(\rho)_{t} \neq \varphi(\tau)_{t}$. Therefore, $\varphi(\tau)_{t} \neq m \varphi(\sigma)_{t}$, and $\nu(\varphi(m \sigma-\tau))=t$.

ii) Assume that $t=\max S_{m}$. If $t=\infty$, then it follows from a) that $\nu(\varphi(n \sigma-\tau))=\infty$. Hence, we can assume: $t<\infty$. Let $n$ be the lowest integer such that $t \in S_{n}$. According to 1$), S_{n}=S_{m}$ and $n$ divides $m$, say $m=p n$. Let $\rho \in H$ such that $\nu(n \sigma-\rho)=t$. One sees that $\nu(m \sigma-p \rho)=t$ and that $\overline{m \sigma-p \rho} \notin H_{\geq t} / H_{>t}$. Indeed, $\nu(m \sigma-p \rho)=\nu(p(n \sigma-\rho)) \geq \nu(n \sigma-\rho)=t$. Since $t$ is a maximal element 
of $S_{m}, \nu(m \sigma-p \rho)=t$, and also $\overline{m \sigma-p \rho} \notin H_{\geq t} / H_{>t}$. By construction, $\varphi(\sigma)_{t}=\frac{1}{n}\left(\overline{n \sigma-\rho}+\varphi(\rho)_{t}\right)$. Hence:

$$
\begin{aligned}
\varphi(\tau-m \sigma)_{t} & =\varphi(\tau)_{t}-m \varphi(\sigma)_{t} \\
& =\varphi(\tau)_{t}-p(\overline{n \sigma-\rho})-p \varphi(\rho)_{t} \\
& =\varphi(\tau)_{t}-p \varphi(\rho)_{t}-p(\overline{n \sigma-\rho}) \notin H_{\geq t} / H_{>t} .
\end{aligned}
$$

It follows: $\varphi(m \sigma-\tau)_{t} \neq 0$. Therefore, $\nu(\varphi(m \sigma-\tau))=t$.

8) As a consequence of 7) b), we have: $\varphi(m \sigma-\tau)=0 \Rightarrow m \sigma-\tau=0$. Thus, $\varphi$ is an isomorphism.

It follows that $(H, \varphi)$ is not a maximal element of $(\mathcal{G}, \leq)$.

Corollary 4.6. Let $(G, \nu)$ be an abelian valued group. Then $\nu$ extends to the divisible hull of $G$.

As a consequence of Theorem 4.5, we can state:

Theorem 6. Let $(G, \nu)$ be an abelian valued group. Let $(\cdot, \cdot, \cdot)$ be the cyclic order defined by $<$ and let $\varpi$ be the Dedekind cut $(\nu(G),<)$. Then, there exists a cyclic valuation $v$ from $\nu(G) \times G$ onto $\nu(G) \cup\{\infty\}$ such that:

$v$ is support-definable,

$\nu$ is $v(\varpi, \cdot)$,

the support of every element of $G$ is well-ordered.

\section{Representation by monomials.}

In this section, we will denote by $(E, O, u, C)$ a cyclically ultrametric space with a fixed element $O$.

Lemma 5.1. Let $(E, O, u, C)$ be a cyclically ultrametric space with a fixed element $O$, and such that $u$ is support-definable.

(a) If $\mu$ is a monomial, then $u(a, \sigma, \mu) \neq \operatorname{deg}(\mu) \Rightarrow u(a, \sigma, \mu) \in \operatorname{Supp}(\sigma, O)$.

(b) Let $\sigma \in E, a \in C$ such that $u(a, \sigma, O)=a$. Then there exists at most one monomial $\mu$ of degree $a$ such that $u(a, \sigma, \mu) \neq a$.

Proof. .

(a) Follows from Lemma 2.2.

(b) Assume that $\mu$ is a monomial of degree $a$ such that $u(a, \sigma, \mu) \neq a$ and let $\mu^{\prime}$ be another monomial. If $\mu^{\prime}$ has not degree $a$, then $a=u(a, \sigma, O) \neq u\left(a, \mu^{\prime}, O\right)$, and $u\left(a, \sigma, \mu^{\prime}\right)=\min _{a}\left(u(a, \sigma, O), u\left(a, \mu^{\prime}, O\right)\right)=a$. If $\mu^{\prime}$ has degree $a$, then, by (a), $u\left(a, \mu, \mu^{\prime}\right)=a \neq u(a, \sigma, \mu)$. Thus $u\left(a, \sigma, \mu^{\prime}\right)=\min _{a}\left(u\left(a, \mu, \mu^{\prime}\right), u(a, \sigma, \mu)\right)=$ $a$.

Lemma 5.2. Let $(E, O, u, C)$ be a cyclically ultrametric space such that the set of monomials u-represents $E$. Let $\sigma$ in $E$ whose support contains at least two elements, and let a in $\operatorname{Supp}(\sigma, O)$. Then:

(a) there exists a unique monomial $\mu_{a, \sigma}$ of degree a such that $u\left(a, \sigma, \mu_{a, \sigma}\right) \neq a$,

(b) $u\left(a, \sigma, \mu_{a, \sigma}\right) \in \operatorname{Supp}(\sigma, O)$,

(c) $\forall c \in \operatorname{Supp}(\sigma, O), c \neq a \Rightarrow c \geq_{a} u\left(a, \sigma, \mu_{a, \sigma}\right)$.

Proof. (a) By assumption, there exists a monomial $\mu_{a, \sigma}$ such that $u\left(a, \sigma, \mu_{a, \sigma}\right) \neq a$. The uniqueness of $\mu_{a, \sigma}$ follows from (b) of Lemma 5.1.

(b) According to (a) of Lemma 5.1, $u\left(a, \sigma, \mu_{a, \sigma}\right) \in \operatorname{Supp}(\sigma, O)$.

(c) Let $c \in C$ such that $a<_{a} c<_{a} u\left(a, \sigma, \mu_{a, \sigma}\right)$. Then $u\left(c, \sigma, \mu_{a, \sigma}\right)=u\left(a, \sigma, \mu_{a, \sigma}\right)$, since $u$ is defined by the supports. Now, $c<_{c} u\left(c, \sigma, \mu_{a, \sigma}\right)<_{c} a=u\left(c, \mu_{a, \sigma}, O\right)$. Hence $u(c, \sigma, O)=u\left(c, \sigma, \mu_{a, \sigma}\right) \neq c$. So $c \notin$ $\operatorname{Supp}(\sigma, O)$.

Definition 5.3. Let $(E, O, u, C)$ be a cyclically ultrametric space such that the set of monomials $u$ represents $E$. Let $a \in C$ and $\sigma \in E$. If $u(a, \sigma, O)=a$, then $\mu_{a, \sigma}$ will denote the only monomial such that $u\left(a, \sigma, \mu_{a, \sigma}\right) \neq a$. If $u(a, \sigma, O) \neq a$, then we set $\mu_{a, \sigma}:=O$. In any case (according to Lemma 5.2), $u\left(a, \sigma, \mu_{a, \sigma}\right)=\min _{a}(\operatorname{Supp}(\sigma, O) \backslash\{a\})$. We will say that $u\left(a, \sigma, \mu_{a, \sigma}\right)$ is the successor of $a$ in $\operatorname{Supp}(\sigma, O)$.

Let $E$ be a subset of the cartesian product $\prod_{a \in C} \Gamma_{a}$ which satisfies condition (*) of Proposition 2.3. We have already proved that $(E, u, C)$ is a cyclically ultrametric space such that $u$ is support-definable.

We fix an element $O$ in $E$ and we assume that $E$ contains all the monomials. Then it is routine to prove that the set of monomials $u$-represents $E$, and for every $a \in C, \Gamma_{a} \simeq M_{a} \cup\{O\}$. 
Theorem 7. Let $(E, O, u, C)$ be a cyclically ultrametric space such that the set of monomials u-represents $E$. For every $\sigma \in E$, set $\varphi(\sigma):=\left(\mu_{a, \sigma}\right)_{a \in C} \in \prod_{a \in C}\left(M_{a} \cup\{O\}\right)$. Then $\varphi$ is an isomorphism from $E$ into a cyclically ultrametric subspace of $\prod_{a \in C}\left(M_{a} \cup\{O\}\right)$ which satisfies condition $\left(^{*}\right)$ of Proposition 2.3.

Proof. In order to prove that $\varphi$ is one-to-one, we let $\sigma \neq \sigma^{\prime}$ in $E$ and we show that $u\left(a, \sigma, \sigma^{\prime}\right)=a \Leftrightarrow$ $\mu_{a, \sigma} \neq \mu_{a, \sigma^{\prime}}$. Assume that $\mu_{a, \sigma} \neq \mu_{a, \sigma^{\prime}}$, then: $u\left(a, \sigma^{\prime}, \mu_{a, \sigma}\right)=a \neq u\left(a, \sigma, \mu_{a, \sigma}\right)$. Hence: $u\left(a, \sigma, \sigma^{\prime}\right)=$ $\min _{a}\left(u\left(a, \sigma, \mu_{a, \sigma}\right), u\left(a, \mu_{a, \sigma}, \sigma^{\prime}\right)=a\right.$. Conversely, if $u\left(a, \sigma, \sigma^{\prime}\right)=a$, then:

$$
a=u\left(a, \sigma, \sigma^{\prime}\right) \geq_{a} \min _{a}\left(u\left(a, \sigma, \mu_{a, \sigma}\right), u\left(a, \mu_{a, \sigma}, \sigma^{\prime}\right)\right) \geq_{a} a .
$$

Thus: $\min _{a}\left(u\left(a, \sigma, \mu_{a, \sigma}\right), u\left(a, \mu_{a, \sigma}, \sigma^{\prime}\right)\right)=a$, so $u\left(a, \sigma^{\prime}, \mu_{a, \sigma}\right)=a$, i.e. $\mu_{a, \sigma} \neq \mu_{a, \sigma^{\prime}}$.

Let $\sigma$ and $\sigma^{\prime}$ in $E, a$ in $C$ and $b=u\left(a, \sigma, \sigma^{\prime}\right)$. So: $\mu_{b, \sigma} \neq \mu_{b, \sigma^{\prime}}$. Now let $c \in C$ such that $\mu_{c, \sigma} \neq \mu_{c, \sigma^{\prime}}$. Then $u\left(c, \sigma, \sigma^{\prime}\right)=c$. By hypothesis, $u$ is defined by the supports, so $c \geq_{a} b$. It follows: $b=\min _{a}\{c \in$ $\left.C \mid \mu_{c, \sigma} \neq \mu_{c, \sigma^{\prime}}\right\}$. Consequently, $\varphi(E)$ satisfies condition $(*)$ of Proposition 2.3, and $\varphi$ is an isomorphism of cyclically ultrametric spaces.

The image of $(E, O, u, C)$ needs not be a subset of any Hahn product. For example, let $C$ be the cyclically ordered set $\mathbb{Z}$. For every $a \in C$, set $\Gamma_{a}:=\{0,1\}$. In the cartesian product $\prod_{a \in C} \Gamma_{a}$, we let $O$ be the element $(0)_{a \in C}$, and $E$ be the subset that consists of all elements whose support is finite and of all elements whose support is equal to $p \mathbb{Z}$, where $p$ is any prime number. The cyclically ultrametric space $(E, O, u, C)$ satisfies condition $(*)$, however it can't be embedded in any Hahn product.

Now let us focus on cyclically valued groups.

Lemma 5.4. Let $(G,+, v)$ be a cyclically valued group such that $v$ is support-definable. Then, for every $a \in C,\left(M_{a} \cup\{0\},+\right)$ is a subgroup of $G$.

Proof. Let $\mu$ be a monomial. According to general properties of valued groups, we have: $\forall c \in C$, $v(c,-\mu)=v(c, \mu)$. Hence $-\mu$ is a monomial, and its degree is equal to the degree of $\mu$. Now, let $\mu_{1}$ and $\mu_{2}$ be monomials of degree $a$. By (b) of Lemma 2.2, it follows that either $\mu_{1}+\mu_{2}=0$, or $\mu_{1}+\mu_{2}$ is a monomial of degree $a$.

Let $(G,+, v)$ be a cyclically valued group such that the set of monomials $v$-represents $G$. For every $a \in C$, let $\varphi_{a}$ be the mapping from $G$ onto $M_{a} \cup\{0\}$ such that, for all $\sigma \in G, \varphi_{a}(\sigma)=\mu_{a, \sigma}$. Then $\varphi_{a}$ is a morphism. Indeed, let $\sigma$ and $\rho$ in $G$, then $v\left(a, \sigma-\rho-\left(\mu_{a, \sigma}-\mu_{a, \rho}\right)\right)=v\left(a, \sigma-\mu_{a, \sigma}-\left(\rho-\mu_{a, \rho}\right)\right) \geq_{a}$ $\min _{a}\left(v\left(a, \sigma-\mu_{a, \sigma}\right), v\left(a, \rho-\mu_{a, \rho}\right)\right)>{ }_{a} a$.

The proof of the following proposition is left to the reader.

Proposition 5.5. Let $\left(\Gamma_{a}\right)_{a \in C}$ be a family of abelian groups, $E$ be a subgroup of $\prod_{a \in C} \Gamma_{a}$ which satisfies condition $\left(^{*}\right)$ of Proposition 2.4. If $E$ contains all the monomials, then the set of monomials v-represents $E$. If this holds, then for every $a \in C, \Gamma_{a} \simeq M_{a} \cup\{O\}$.

For every cyclically valued group $G$ such that the set of monomials $v$-represents $G$, and for every $\sigma \in G$, we set $\varphi(\sigma):=\left(\mu_{a, \sigma}\right)_{a \in C} \in \prod_{a \in C}\left(M_{a} \cup\{0\}\right)$.

Theorem 8. Let $G$ be a cyclically valued group such that the set of monomials v-represents $G$. Then $\varphi$ is an isomorphism from $G$ into a subgroup of $\prod_{a \in C}\left(M_{a} \cup\{0\}\right)$ which satisfies condition (*) of Proposition 2.4. If this holds, then for every $a \in C, \Gamma_{a} \simeq \prod_{a \in C}\left(M_{a} \cup\{0\}\right)$.

Proof. Left to the reader.

In some cases, we can refine Theorem 5 in such a way that the set of monomials $u$-represent $E$.

Proposition 5.6. Let $(E, \nu)$ be an ultrametric space, $T:=\nu(E, E), O$ in $E$ and $(\cdot, \cdot, \cdot)$ be the cyclic order defined by the order $<$. Then the following (1) and (2) are equivalent.

(1) For every $t \in T$ and for every $\sigma \in E$, the number of open balls of radius $t$ contained in $B_{c}(\sigma, t)$ is lower than (or equal to) the number of open balls of radius $t$ contained in $B_{c}(O, t)$.

(2) There exists a cyclic ultrametric distance $u$ on $(E, O)$ such that the support of every element is well-ordered and the set of monomials u-represents E. Furthermore, if $\varpi$ is the Dedekind cut $(T,<)$ of $(T,(\cdot, \cdot, \cdot))$, then $u(\varpi, \cdot, \cdot)$ is equal to $\nu$.

If (1), (2) hold, then for every $t$ in $T$, the number of monomials of degree $t$ is equal to the number of open balls of radius $t$ contained in $B_{c}(O, t)$. 
Proof. .

The implication $(1) \Rightarrow(2)$ is a consequence of Theorem 5. Indeed, Trivially, $u$ is support-definable. Now, by assumption, for every $t \in T, M_{t}$ is a set of representatives of the elements of $\mathcal{B}(O, t) \backslash\left\{B_{o}(O, t)\right\}$. We let $f$ be the embedding from $E$ into $\sqcap_{t \in T}\left(\{O\} \cup M_{t}\right)$. Recall that for every $t \in T$ and $\rho \in M_{t}, f(\rho)_{t}=\rho$ and, if $t^{\prime} \neq t, f(\rho)_{t^{\prime}}=O$. We set, for every $t$ in $T, \sigma, \tau$ in $E, u(t, \sigma, \tau):=\min \left\{t^{\prime} \in \operatorname{Supp}(f(\sigma), f(\tau)) \mid t \leq t^{\prime}\right\}$. So the set of all monomials of degree $t$ is $M_{t}$. Now let $\sigma \in E$ and assume that $f(\sigma)=\left(\rho_{t}\right)_{t \in T}$, with $\rho_{t} \in M_{t} \subset E$. Then, for all $t \in T, u\left(t, \sigma, \rho_{t}\right)=\min \left\{t^{\prime} \in \operatorname{Supp}\left(f(\sigma), f\left(\rho_{t}\right)\right) \mid t \leq t^{\prime}\right\}>t$. Therefore the set of monomials $u$-represents $E$.

Assume that (2) holds. For every $t$ in $T$, let $M_{t}$ be the set of all monomials of degree $t$. Let $\sigma \in E$, $t \in T, \tau, \tau^{\prime}$ in $B_{c}(\sigma, t)$. We have $\nu\left(\tau, \tau^{\prime}\right) \geq \min \left(\nu\left(\tau, \tau^{\prime}\right), \nu\left(\sigma, \tau^{\prime}\right)\right) \geq t$, hence $u\left(t, \tau, \tau^{\prime}\right)=\nu\left(\tau, \tau^{\prime}\right)$. Now,

$$
\begin{aligned}
B_{o}(\tau, t)=B_{o}\left(\tau^{\prime}, t\right) & \Leftrightarrow \nu\left(\tau, \tau^{\prime}\right)>t \\
& \Leftrightarrow u\left(t, \tau, \tau^{\prime}\right)>t \\
& \Leftrightarrow \mu_{t, \tau}=\mu_{t, \tau^{\prime}} .
\end{aligned}
$$

Hence we can define a one-to-one mapping $\phi$ from $\mathcal{B}(\sigma, t)$ into $M_{t} \cup\{O\}$, by setting, for every $B \in \mathcal{B}(\sigma, t)$, $\phi(B):=\mu_{t, \tau}$, with $\tau$ any element of $B$. Now, in the same way, there is a one-to-one mapping from $\mathcal{B}(O, t)$ onto $\{O\} \cup M_{t}$.

\section{REFERENCES}

[C-K 73] Chang, C.C. \& Keisler, H. J. Model Theory, North-Holland, Amsterdam, 1973

[Co 53] Conrad, P. F. Embedding theorems for abelian groups with valuations, Amer. J. Math. 75 (1953), 1-29.

[D 84] Delon, F. Espaces ultramétriques, J. of Symbolic Logic 49 (1984), 405-424.

[Fu 63] Fuchs, L. Partially Ordered Algebraic Structures, Pergamon Press, 1963.

[GKL] Giraudet, M. Kuhlmann, F.-V. \& Leloup, G. Formal power series with cyclically ordered exponents, to appear in Arkiv der Mathematiks.

[Ku 45] Kulikov, L. Ya. On the theory of abelian groups of arbitrary cardinality, Math. Sb. 16 (1945), 129-162.

[No 82] Novák, V. Cyclically ordered sets, Czech. Math. J. 32 (1982), 460-473.

[No 84] Novák, V. Cuts in cyclically ordered sets, Czech. Math. J. 34 (1984), 322-333.

[Nos 87] Novák, V. \& Novotný, M. On completions of cyclically ordered sets, Czech. Math. J. 37 (1987), $407-414$.

[P-R 93] Priess-Crampe, S. \& Ribenboim, P. Fix Points, combs and generalized power series, Abh. Math. Sem. Univ. Hamburg 63 (1993), 227-244.

[P-R 96] Priess-Crampe S. \& Ribenboim, P. Generalized ultrametric spaces, Abh. Math. Sem. Univ. Hamburg 66 (1996), 55-73.

[Ri 64] Ribenboim, P. Théorie des Valuations, Les Presses de l'Université de Montréal, Montréal, 1964.

U.P.R.E.S.A. 7056 (Equipe de Logique, Paris ViI), and, Département de Mathématiques, Faculté des Sciences, avenue Olivier Messiaen, 72085 LE MANS CEDEX, FRANCE

E-mail address: leloup_At_logique.jussieu.fr 